\title{
Uncovering Non-Fermi-Liquid Behavior in Hund Metals: Conformal Field Theory Analysis of an $\mathrm{SU}(2) \times \mathrm{SU}(3)$ Spin-Orbital Kondo Model
}

\author{
E. Walter $\odot,{ }^{1}$ K. M. Stadler $\odot,{ }^{1}$ S.-S. B. Lee $\odot,{ }^{1}$ Y. Wang $\odot,{ }^{2}$ G. Kotliar, ${ }^{2,3}$ A. Weichselbaum, ${ }^{2,1}$ and J. von Delft ${ }^{1}$ \\ ${ }^{1}$ Arnold Sommerfeld Center for Theoretical Physics, Center for NanoScience, \\ and Munich Center for Quantum Science and Technology, \\ Ludwig-Maximilians-Universität München, 80333 Munich, Germany \\ ${ }^{2}$ Condensed Matter Physics and Materials Science Department, Brookhaven National Laboratory, \\ Upton, New York 11973, USA \\ ${ }^{3}$ Department of Physics and Astronomy, Rutgers University, Piscataway, New Jersey 08854, USA
}

(Received 12 August 2019; revised 27 May 2020; accepted 7 July 2020; published 3 September 2020)

Hund metals have attracted attention in recent years due to their unconventional superconductivity, which supposedly originates from non-Fermi-liquid (NFL) properties of the normal state. When studying Hund metals using dynamical mean-field theory, one arrives at a self-consistent "Hund impurity problem" involving a multiorbital quantum impurity with nonzero Hund coupling interacting with a metallic bath. If its spin and orbital degrees of freedom are screened at different energy scales, $T_{\mathrm{sp}}<T_{\text {orb }}$, the intermediate energy window is governed by a novel NFL fixed point, whose nature had not yet been clarified. We resolve this problem by providing an analytical solution of a paradigmatic example of a Hund impurity problem, involving two spin and three orbital degrees of freedom. To this end, we combine a state-ofthe-art implementation of the numerical renormalization group, capable of exploiting non-Abelian symmetries, with a generalization of Affleck and Ludwig's conformal field theory (CFT) approach for multichannel Kondo models. We characterize the NFL fixed point of Hund metals in detail for a Kondo model with an impurity forming an $\mathrm{SU}(2) \times \mathrm{SU}(3)$ spin-orbital multiplet, tuned such that the NFL energy window is very wide. The impurity's spin and orbital susceptibilities then exhibit striking power-law behavior, which we explain using CFT arguments. We find excellent agreement between CFT predictions and numerical renormalization group results. Our main physical conclusion is that the regime of spinorbital separation, where orbital degrees of freedom have been screened but spin degrees of freedom have not, features anomalously strong local spin fluctuations: the impurity susceptibility increases as $\chi_{\mathrm{sp}}^{\mathrm{imp}} \sim \omega^{-\gamma}$, with $\gamma>1$.

DOI: $10.1103 /$ PhysRevX.10.031052

\section{INTRODUCTION}

\section{A. Motivation: Hund metals}

Hund metals are multiorbital materials with broad bands which are correlated via the ferromagnetic Hund coupling $J_{H}$, rather than the Hubbard interaction $U$. The coupling $J_{H}$ implements Hund's rule, favoring electronic states with maximal spin, which causes Hund metals to be fundamentally different from Mott insulators. This is a new exciting area of condensed matter physics; for a recent review with numerous references, see Ref. [1]. Hund metals are a very diverse class of materials, including transition metal oxides

Published by the American Physical Society under the terms of the Creative Commons Attribution 4.0 International license. Further distribution of this work must maintain attribution to the author(s) and the published article's title, journal citation, and DOI.
Subject Areas: Condensed Matter Physics, Mesoscopics, Strongly Correlated Materials with partially filled $d$ shells, such as the iron-based pnictide and selenide superconductors, the ruthenates, and many others [1-13].

The iron-based superconductors, in particular, raised much interest in recent years because of the unconventional nature of their superconductivity. It has been argued that the Hund nature of their normal state is essential for the onset of superconductivity [14]. In particular, spin fluctuations with a power-law divergent susceptibility $\propto \omega^{-\gamma}$, with $\gamma>1$, have been evoked in an explanation for the anomalously large ratio of $2 \Delta_{\max } / T_{c}$ observed experimentally, where $\Delta_{\max }$ is the maximum superconducting gap and $T_{c}$ the critical temperature [14]. The normal state of Hund metals is of great interest on its own, since it typically shows bad-metal behavior [6,15,16]. Motivated by these considerations, computational and experimental studies of Hund metals have begun to uncover their rich physics in recent years [4,5,8,11,12,17-21]. 
When studying Hund metals in the context of dynamical mean-field theory (DMFT), the problem of a crystal lattice with many strongly interacting lattice sites is mapped onto a "Hund impurity," coupled self-consistently to an effective noninteracting metallic bath. A Hund impurity has both spin and orbital degrees of freedom and a finite Hund coupling, favoring a large local spin.

A particularly fascinating consequence of the interplay between spin and orbital degrees of freedom is the phenomenon of spin-orbital separation (SOS): Kondo screening of Hund impurity models occurs in two stages, and the energy scales below which free spin and orbital degrees are screened differ, $T_{\mathrm{sp}}<T_{\text {orb }}$ [8,9,22-24]. The low-energy regime below $T_{\mathrm{sp}}$ shows Fermi-liquid (FL) behavior. The intermediate SOS window $\left[T_{\mathrm{sp}}, T_{\text {orb }}\right]$, by contrast, shows incoherent behavior, featuring almost fully screened orbital degrees of freedom coupled to almost free spin degrees of freedom. The incoherent regime has been conjectured to have non-Fermi-liquid (NFL) properties and argued to be relevant for the bad-metal behavior of Hund metals $[8,25]$. However, the nature of the putative underlying NFL state has not yet been clarified.

A major obstacle for analyzing the conjectured NFL regime of Hund metals has been a lack of detailed, analytical understanding of the basic properties of Hund impurity models, since theoretical work has overwhelmingly focused on Kondo models without orbital degrees of freedom. In this work, we overcome this obstacle in the context of an instructive case study of a specific Hund impurity model.

Before specifying the latter in detail, though, let us put our study into perspective by providing a brief historical overview of Hund impurity models.

\section{B. Brief history of Hund impurity models}

Hund impurity models are natural multiorbital generalizations of single-orbital magnetic impurity models such as the Kondo model used by Kondo in 1964 to explain the resistance minimum in magnetic alloys [26]. The search for a detailed understanding of the Kondo model beyond Kondo's perturbative calculation was a cornerstone toward the development of renormalization group techniques, starting with Anderson's poor man's scaling approach [27] and culminating in Wilson's numerical renormalization group (NRG) [28]. These methods confirmed that below a characteristic Kondo temperature the metallic bath screens the impurity spin, leading to the formation of a singlet state between impurity and conduction electrons.

Following these findings, naturally the question arises: What happens if the impurity has multiple orbitals? In particular, electrons on a multiorbital impurity experience not only a Coulomb interaction stabilizing a magnetic moment on the impurity, but also a Hund coupling, enforcing the effect of Hund's rule to maximize the total impurity spin. These two interactions lead to an intricate interplay, crucially depending on the number of electrons on the impurity. Indeed, it had been observed already in the 1960s that the Kondo scale for impurities in transition metal alloys with partially filled $d$ shells decreases exponentially as the shell filling approaches 1/2 [29,30], drawing attention to the question of understanding Kondo screening in the presence of multiple orbitals. Coqblin and Schrieffer [31] developed a generalization of the Kondo model for multiorbital impurities, yet only involving the spin degree of freedom. Okada and Yosida [32] included orbital degrees of freedom and in particular pointed out the importance of a finite Hund coupling, enforcing the effect of Hund's rule in such multiorbital systems. However, theoretical tools for analyzing a model with nonzero Hund coupling away from half filling were lacking at the time.

Later, Nozières and Blandin [33] studied a spin Kondo impurity immersed in a metallic bath with multiple orbital channels. A major conclusion of their work was that such models lead to overscreening of the impurity spin and NFL behavior, if the number of channels exceeds twice the impurity spin $(k>2 S)$. This generated great theoretical interest in multichannel Kondo models, including exact Bethe solutions providing information on thermodynamical properties [34-39], and NRG studies [40,41]. Affleck and Ludwig (AL) [42-46] developed a powerful conformal field theory (CFT) approach for studying the strongcoupling fixed points of such multiband Kondo models, providing analytical results for finite-size spectra and the scaling behavior of correlation functions. However, their work was restricted to pure spin impurities without nontrivial orbital structure. Thus, their methods have not yet been applied to Hund impurity models, including orbital degrees of freedom and a finite Hund coupling.

In this work, we fill this long-standing void and provide a detailed and comprehensive analysis of a prototypical Hund impurity model (specified below). We achieve this by advancing and combining two powerful complementary techniques that both arose in the very context of Kondo physics: An analytical solution based on AL's celebrated CFT approach, generalized from a pure spin impurity to one with spin and orbital structure, and a quasiexact numerical solution using a state-of-the-art implementation of Wilson's NRG, allowing studies of multiorbital systems by fully exploiting Abelian and non-Abelian symmetries. This allows us to achieve a detailed understanding of the NFL behavior arising in this Hund impurity model.

\section{Minimal models for Hund metals}

We next describe the considerations motivating the specific choice of model studied below.

A minimal model for Hund metals has been proposed in Ref. [8]. It is a three-orbital Hubbard-Hund model, and it has been studied extensively in Refs. [2,6,9,11,22-24,47]. 
A treatment of this model by DMFT at $1 / 3$ filling yields a self-consistent Hund impurity model. More specifically, one obtains a self-consistent three-orbital Anderson-Hund (3oAH) model, in which bath and impurity both have spin and orbital degrees of freedom. The impurity hosts two electrons forming an antisymmetric orbital triplet and a symmetric spin triplet $(S=1)$, reflecting Hund's rule. At energies so low that charge fluctuations can be treated by a Schrieffer-Wolff transformation [9], the 3oAH model maps onto a three-channel spin-orbital Kondo $(3 \mathrm{soK})$ model whose impurity forms a $(3 \times 3)$-dimensional $\mathrm{SU}(2) \times \mathrm{SU}(3)$ spin-orbital multiplet.

The 3oAH model exhibits SOS [8,9,22-24]. Within the SOS window $\left[T_{\mathrm{sp}}, T_{\mathrm{orb}}\right]$, the imaginary part of the spin susceptibility scales as $\chi_{\mathrm{sp}}^{\mathrm{imp}} \sim \omega^{-6 / 5}[22,47]$. The fact that the exponent, $\gamma=6 / 5$, is larger than 1 has been argued to lead to the anomalous superconducting state of the iron pnictide Hund metals, as mentioned above [14]. However, the origin of this power law has remained unclear. One impediment toward finding an explanation is the fact that for the 3oAH model the orbital and spin screening scales cannot be tuned independently. The SOS window turns out to be rather small, masking the NFL behavior expected to occur within it.

In this paper, we sidestep this limitation by instead studying the 3 soK model and treating its exchange couplings as independent parameters, freed from the shackles of their 3oAH origin. We tune these such that the regime of SOS is very wide, with $T_{\text {sp }} \ll T_{\text {orb }}$. This enables us to characterize the NFL fixed point obtained for $T_{\mathrm{sp}}=0$, which also governs the intermediate NFL window if $T_{\text {sp }} \ll T_{\text {orb }}$. We compute fixed-point spectra and the scaling behavior of dynamical spin and orbital susceptibilities using both NRG and CFT, with mutually consistent results. In particular, we find an analytical explanation for the peculiar power law $\chi_{\mathrm{sp}}^{\mathrm{imp}} \sim \omega^{-6 / 5}$ : It turns out to be governed (albeit somewhat indirectly) by the NFL fixed point mentioned above. Finally, we demonstrate the relevance of these 3 soK results for the low-energy behavior of the 3oAH model by employing a hybrid Anderson-Kondo model which smoothly interpolates between the physics of the $3 \mathrm{soK}$ and $3 \mathrm{oAH}$ models. This interpolation shows that our new results also shed light on previous DMFT results for a self-consistent $3 \mathrm{oAH}$ model $[22,47]$.

Our CFT analysis builds on that devised by AL [42-46] for the $k$-channel Kondo model, describing $k$ spinful channels exchange coupled to an impurity with spin $S$, but no orbital degrees of freedom. If $k>2 S$, the impurity spin is overscreened. AL described the corresponding NFL fixed point using a charge-spin-orbital $\mathrm{U}(1) \times \mathrm{SU}(2)_{k} \times$ $\mathrm{SU}(\mathrm{k})_{2}$ Kac-Moody (KM) decomposition of the bath states, and fusing the spin degrees of freedom of impurity and bath using $\mathrm{SU}(2)_{k}$ fusion rules. Here we generalize this strategy to our situation, where the impurity has spin and orbital "isospin" degrees of freedom: the NFL fixed point at
$T_{\mathrm{sp}}=0$ can be understood by applying $\mathrm{SU}(3)_{2}$ fusion rules in the orbital sector, leading to orbital overscreening. If $T_{\mathrm{sp}}$ is nonzero (but $\ll T_{\text {orb }}$ ), the overscreened orbital degrees of freedom couple weakly to the impurity spin, driving the system to a FL fixed point. There both spin and orbital degrees of freedom are fully screened, in a manner governed by $\mathrm{SU}(6)_{1}$ fusion rules.

The paper is structured as follows. Section II defines the 3 soK model and discusses its weak-coupling renormalization group (RG) flow. Section III presents our NRG results. Section IV gives a synopsis of our CFT results, summarizing all essential insights and arguments, while Sec. V elaborates the corresponding CFT arguments in more detail. Section VI discusses a hybrid Anderson-Kondo model which interpolates between the 3 soK model and the 3oAH model. Section VII summarizes our conclusions. The Appendix revisits a two-channel spin-orbital Kondo model studied by Ye in 1997 [48], pointing out the similarities and differences between his work and ours.

\section{MODEL, PERTURBATIVE RG FLOW}

We study the 3 soK model proposed in Ref. [9]. $H_{\text {bath }}=$ $\sum_{p m \sigma} \varepsilon_{p} \psi_{p m \sigma}^{\dagger} \psi_{p m \sigma}$ describes a symmetric, flat-band bath, where $\psi_{p m \sigma}^{\dagger}$ creates an electron with momentum $p$ and spin $\sigma$ in orbital $m \in\{1,2,3\}$. The bath couples to the impurity spin $\mathbf{S}$ and orbital isospin $\mathbf{T}$ via

$$
H_{\text {int }}=J_{0} \mathbf{S} \cdot \mathbf{J}_{\mathrm{sp}}+K_{0} \mathbf{T} \cdot \mathbf{J}_{\text {orb }}+I_{0} \mathbf{S} \cdot \mathbf{J}_{\mathrm{sp}-\text { orb }} \cdot \mathbf{T} .
$$

Here $\mathbf{S}$ are $\mathrm{SU}(2)$ generators in the $S=1$ representation, normalized as $\operatorname{Tr}\left(S^{\alpha} S^{\beta}\right)=\frac{1}{2} \delta^{\alpha \beta}$, and $\mathbf{T}$ are $\mathrm{SU}(3)$ generators in the representation with Young diagram $\boxminus$, and $\operatorname{Tr}\left(T^{a} T^{b}\right)=\frac{1}{2} \delta^{a b} . \mathbf{J}_{\mathrm{sp}}, \mathbf{J}_{\text {orb }}$, and $\mathbf{J}_{\text {sp-orb }}$ are the bath spin, orbital, and spin-orbital densities at the impurity site, with $J_{\mathrm{sp}}^{\alpha}=\psi_{m \sigma}^{\dagger} \frac{1}{2} \sigma_{\sigma \sigma^{\prime}}^{\alpha} \psi_{m \sigma^{\prime}}, J_{\mathrm{orb}}^{a}=\psi_{m \sigma}^{\dagger} \frac{1}{2} \tau_{m m^{\prime}}^{a} \psi_{m^{\prime} \sigma}, J_{\mathrm{sp}-\text { orb }}^{\alpha, a}=$ $\psi_{m \sigma}^{\dagger} \frac{1}{2} \sigma_{\sigma \sigma^{\prime}}^{\alpha} \frac{1}{2} \tau_{m m^{\prime}}^{a} \psi_{m^{\prime} \sigma^{\prime}}$ (summation over repeated indices is implied), where fields are evaluated at the impurity site, $\psi_{m \sigma}^{\dagger}(r=0)$, and $\sigma^{\alpha}\left[\tau^{a}\right]$ are Pauli [Gell-Mann] matrices, with normalization $\operatorname{Tr}\left(\sigma^{\alpha} \sigma^{\beta}\right)=2 \delta^{\alpha \beta}\left[\operatorname{Tr}\left(\tau^{a} \tau^{b}\right)=2 \delta^{a b}\right]$. We use Young diagrams as labels for irreducible representations (irreps) of the $\mathrm{SU}(3)$ group. An alternative notation, also frequently used, would be to label SU(3) irreps by their dimension: $\bullet=1, \square=3, \boxminus=\overline{3}$, where $\overline{3}$ refers to the conjugate represenation of $3, \square=6, \square=\overline{6}, \square=8$, etc.

The Hamiltonian has $\mathrm{U}(1)_{\mathrm{ch}} \times \mathrm{SU}(2)_{\mathrm{sp}} \times \mathrm{SU}(3)_{\text {orb }}$ symmetry. We label its symmetry multiplets by $Q=(q, S, \lambda)$, with $q$ the bath particle number relative to half filling (the 3 soK impurity has no charge dynamics; hence we may choose $q_{\mathrm{imp}}=0$ ), $S$ the total spin, and $\lambda$ a Young diagram denoting an $\mathrm{SU}(3)$ representation. The values of the spin, orbital, and spin-orbital exchange couplings, $J_{0}, K_{0}, I_{0}$, can be derived from the 3oAH model by a Schrieffer-Wolff transformation [9]. When the 3oAH model is studied in the 
regime relevant for Hund metals, i.e., with a ferromagnetic on-site Hund coupling $J_{H}$ favoring maximization of the local spin, and with a local filling $n_{d}$ differing by $\simeq 1$ from half filling, the resulting 3 soK exchange couplings $J_{0}, K_{0}, I_{0}$ are typically all positive, i.e., antiferromagnetic. [This can be inferred from Eqs. (4)-(7) of Ref. [9].] Furthermore, when the weak-coupling RG flow of the 3 soK model is studied in the presence of finite $K_{0}>0$ and $I_{0}>0$, one finds that $J_{0}$ flows toward positive values regardless of whether its initial value is chosen positive or negative [the latter case is illustrated by the purple arrows in Fig. 1(a)]. Hence, we here focus on positive exchange couplings only. However, instead of using values obtained from a Schrieffer-Wolff transformation, here we take the liberty of choosing $J_{0}, K_{0}$, $I_{0}$ to be independent, tuning them such that $T_{\mathrm{sp}} \ll T_{\text {orb }}$. This is in extension of the $3 \mathrm{oAH}$ model, in which $T_{\mathrm{sp}}$ is only at most about an order of magnitude smaller than $T_{\text {orb }}$.

Aron and Kotliar [9] have performed a perturbative analysis of the RG flow of the 3 soK model. Their Eqs. (8)-(10) describe the flow of the coupling vector, $\mathbf{c}(D)=(J, K, I)$, upon reducing the half-bandwidth $D$ starting from $\mathbf{c}_{0}=\left(J_{0}, K_{0}, I_{0}\right)$ at $D_{0}$. For the 3 soK model, these equations read

$$
\begin{aligned}
& \beta_{J}=-\left(1-\frac{3}{2} J\right)\left(J^{2}+\frac{2}{9} I^{2}\right)+\cdots, \\
& \beta_{K}=-\frac{3}{2}(1-K)\left(K^{2}+\frac{1}{2} I^{2}\right)+\cdots, \\
& \beta_{I}=-\frac{3}{2}\left[\left(\frac{4}{3} J+2 K-J^{2}-K^{2}\right) I-\frac{5}{18} I^{2}-\frac{17}{36} I^{3}\right]+\cdots,
\end{aligned}
$$

where $\beta_{J}=d J / d \ln D$, etc., with energies in units of $D_{0}$. Figure 1 illustrates the resulting RG flow. There are several fixed points. The free-impurity fixed point, $\mathbf{c}_{\mathrm{FI}}^{*}=(0,0,0)$, is unstable: for any nonzero $\mathbf{c}_{0}$, one or more couplings flow toward strong coupling, and the $D$ values where $J$ or $K$ become of order unity yield estimates of $T_{\mathrm{sp}}$ and $T_{\mathrm{orb}}$, respectively. For $\mathbf{c}_{0}=\left(0, K_{0} \neq 0,0\right)$ [black arrows in Fig. 1(a)], the system flows toward a NFL fixed point, $\mathbf{c}_{\mathrm{NFL}}^{*}=(0,1,0)$. This fixed point is unstable against nonzero $J_{0}$ or $I_{0}$. For $I_{0}=0$, the flow equations for $J$ and $K$ are decoupled, such that for a small but nonzero $J_{0} \ll K_{0}$ (red arrows) the flow first closely approaches $\mathbf{c}_{\mathrm{NFL}}^{*}$, until $J$ grows large, driving it toward a FL fixed point $\mathbf{c}_{\mathrm{FL}}^{*}$. Figure 1(b) shows that the NFL regime $(J \ll K)$ governed by $\mathbf{c}_{\mathrm{NFL}}^{*}$ can be large. For $I_{0} \neq 0$, the $J$ and $K$ flows are coupled, hence the growth of $K$ triggers that of $J$, accelerating the flow toward $\mathbf{c}_{\mathrm{FL}}^{*}$. In this case, the NFL energy window is rather small [cf. Fig. 1(c)]. For example, for $\mathbf{c}_{0}=(0.1,0.3,0.5)$ (light green arrows), typical for the values obtained through a Schrieffer-Wolff 3oAH to 3soK mapping, the RG flow does not approach $\mathbf{c}_{\mathrm{NFL}}^{*}$ very closely; thus fully developed NFL behavior is not observed.
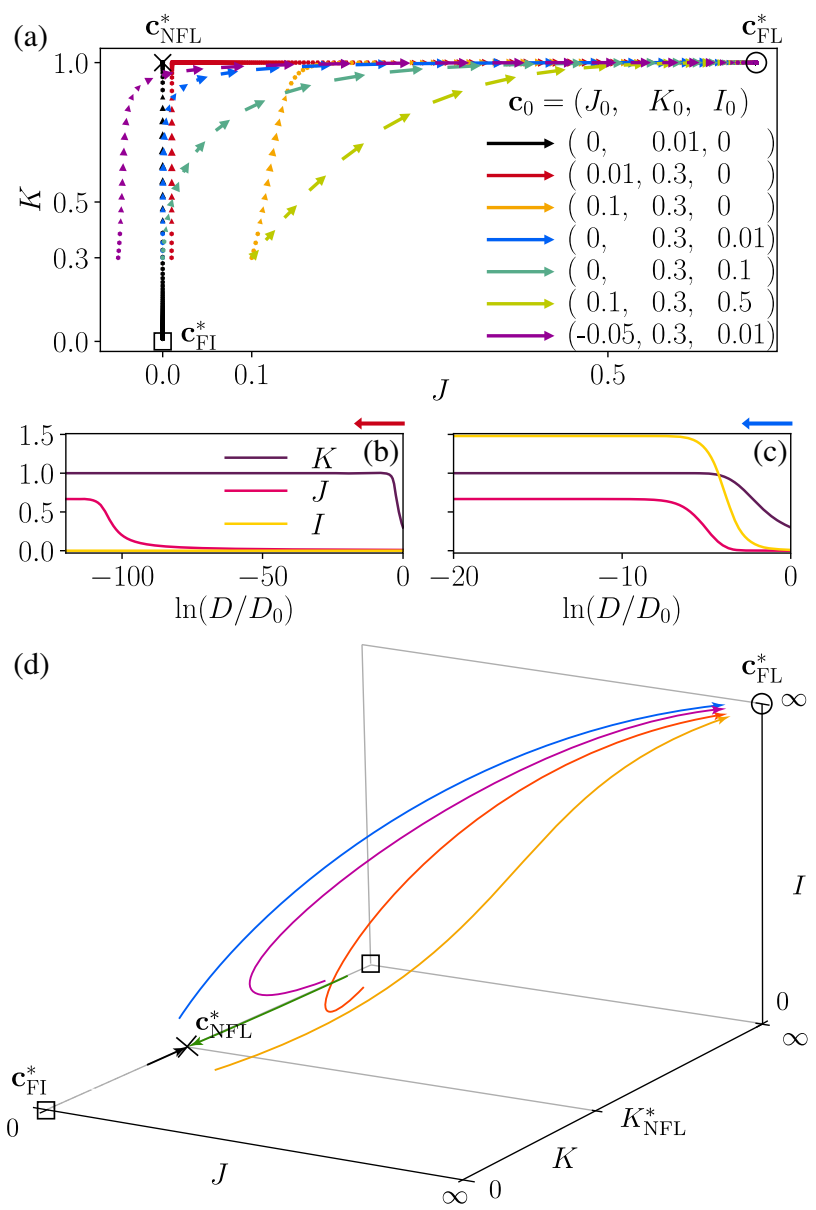

FIG. 1. (a) RG flow of the coupling vector $\mathbf{c}=(J, K, I)$ (projected into the $J-K$ plane), obtained by solving the weakcoupling RG equations (2) [Eqs. (8)-(10) of Ref. [9] ] for various initial values, $\mathbf{c}_{0}=\left(J_{0}, K_{0}, I_{0}\right)$. Arrows depict the gradient vector $-[d /(d \ln D)](J, K)$ at equal steps of $\ln D$. (b),(c) Weak-coupling $\mathrm{RG}$ flow of $\mathbf{c}(D)$ for (b) $\mathbf{c}_{0}=(0.01,0.3,0)$ [red arrows in (a)] and (c) $(0,0.3,0.01)$ [blue arrows in (a)]. (d) Qualitative depiction of the conjectured RG flow in the full $J-K-I$ space, for all couplings non-negative. Fat, faint dashed lines show the solutions c $(D)$ of the weak-coupling equations (2), initialized at $K_{0} \ll$ $K_{\mathrm{NFL}}^{*}$ with $\left(J_{0}, I_{0}\right)=(0,0)$ (black), $(>0,0)$ (yellow), or $(0,>0)$ (blue), and plotted only in the weak-coupling regime [beyond the latter, Eqs. (2) lose validity]. Solid lines, drawn by hand, qualitatively show the flow expected beyond the weak-coupling regime, including trajectories initialized at $K_{0} \gg K_{\mathrm{NFL}}^{*}$, with $\left(J_{0}, I_{0}\right)=(0,0)$ (green), $(>0,0)$ (orange), or $(0,>0)$ (purple). The black squares, cross, and circle depict fixed points.

Figure 1(d) offers a qualitative depiction of the conjectured RG flow in the full $J-K-I$ space, for all couplings nonnegative. Fat, faint dashed lines show the solutions $\mathbf{c}(D)$ of the weak-coupling Eqs. (2). However, these equations lose validity once the couplings are no longer small (and their above-mentioned predictions that $K_{\mathrm{NFL}}^{*}=K_{\mathrm{FL}}^{*}=1$ should not be trusted). Solid lines, drawn by hand, qualitatively depict the flow expected beyond the weakcoupling regime, based on the following considerations. 
First, for $K_{0}>0$ and $J_{0}=I_{0}=0$, the NRG analysis of Sec. III suggests that the flow proceeds along a trajectory where $I$ and $J$ remain zero, reaching a NFL fixed point, $\mathbf{c}_{\mathrm{NFL}}^{*}=\left(0, K_{\mathrm{NFL}}^{*}, 0\right)$ at a finite value of $K_{\mathrm{NFL}}^{*}$. This fixed point is stable, approached by RG flow both from below and above. Correspondingly, the line $J_{0}=I_{0}=0$ contains another fixed point at $K_{0}=\infty$, which is unstable. To understand the latter point heuristically, consider taking $K_{0}$ very large. Then the system will attempt to screen its local orbital degree of freedom, with representation $\boxminus$, into an orbital singlet. Doing so by binding just a bath single electron, spin up or down, would break spin symmetry. Hence, it must bind two bath electrons, spin up and down, yielding a local orbital degree of freedom yet again, with representation $\square$. Thus, choosing $K_{0}$ very large is equivalent to initializing the model with local orbital representation $\square$ and small initial coupling (presumably $\sim 1 / K_{0}$ ). This would grow under the RG flow; hence $K_{0}=\infty$ is an unstable fixed point, just as $K_{0}=0$. (This argumentation is entirely analogous to that familiar from the two-channel Kondo model [33]; for the present 3 soK model, it is further elaborated in Ref. [49].)

For $K_{0}>0$ and $J_{0}, I_{0}$ both non-negative but not both zero, the NRG analysis of Sec. V E suggests that the flow always ends up at a unique FL fixed point $\mathbf{c}_{\mathrm{FL}}^{*}$. Hence $\mathbf{c}_{\mathrm{NFL}}^{*}$ is unstable against turning on $J_{0}$ or $I_{0}$. The fixed point $\mathbf{c}_{\mathrm{FL}}^{*}$ features a fully screened spin and orbital singlet ground state and an excitation spectrum with SU(6) symmetry. This implies that as the flow approaches $\mathbf{c}_{\mathrm{FL}}^{*}$, all three couplings $J, K$, and $I$ tend to infinity, with relative values such that the fixed-point Hamiltonian has SU(6) symmetry, i.e., $3 J=$ $2 K=I[9]$.

\section{NRG RESULTS}

To study the RG flow in a quantitatively reliable manner, we solve the 3 soK model using NRG [28,50,51], exploiting non-Abelian symmetries using QSpace [50]. The bath is discretized logarithmically and mapped to a semiinfinite "Wilson chain" with exponentially decaying hoppings, and the impurity coupled to site 0 . The chain is diagonalized iteratively while discarding high-energy states, thereby zooming in on low-energy properties: the (finite-size) level spacing of a chain ending at site $k$ is of order $\omega_{k} \propto \Lambda^{-k / 2}$, where $\Lambda>1$ is a discretization parameter. The RG flow can be visualized using NRG eigenlevel spectra, showing how the chain's lowest-lying eigenenergies $\mathcal{E}$ evolve when $k$ is increased by plotting the dimensionless rescaled energies $E=\left(\mathcal{E}-\mathcal{E}_{\text {ref }}\right) / \omega_{k}$ versus $\omega_{k}$ for odd $k$. The $E$-level flow is stationary ( $\omega_{k}$ independent) while $\omega_{k}$ traverses an energy regime governed by one of the system's fixed points, but changes during crossovers between fixed points.

$$
\begin{array}{cll}
-\left(+1, \frac{1}{2}, \square\right) & --\left(+3, \frac{1}{2}, \boxminus\right) & -\left(-3, \frac{1}{2}, \boxminus\right), \rightarrow \tilde{\Psi}_{\mathrm{sp}} \\
-\left(+1, \frac{3}{2}, \bullet\right) & --\left(+1, \frac{1}{2}, \bullet\right) & --(0,0, \boxminus), \rightarrow \boldsymbol{\Phi}_{\mathrm{sp}} \\
-(0,0, \square) & -(+2,0, \square) & -\left(-1, \frac{1}{2}, \square\right), \rightarrow \tilde{\Psi}_{\mathrm{orb}} \\
-(-2,0, \bullet) & -(+2,1, \square) & -(0,1, \boxminus), \rightarrow \boldsymbol{\Phi}_{\mathrm{orb}}
\end{array}
$$
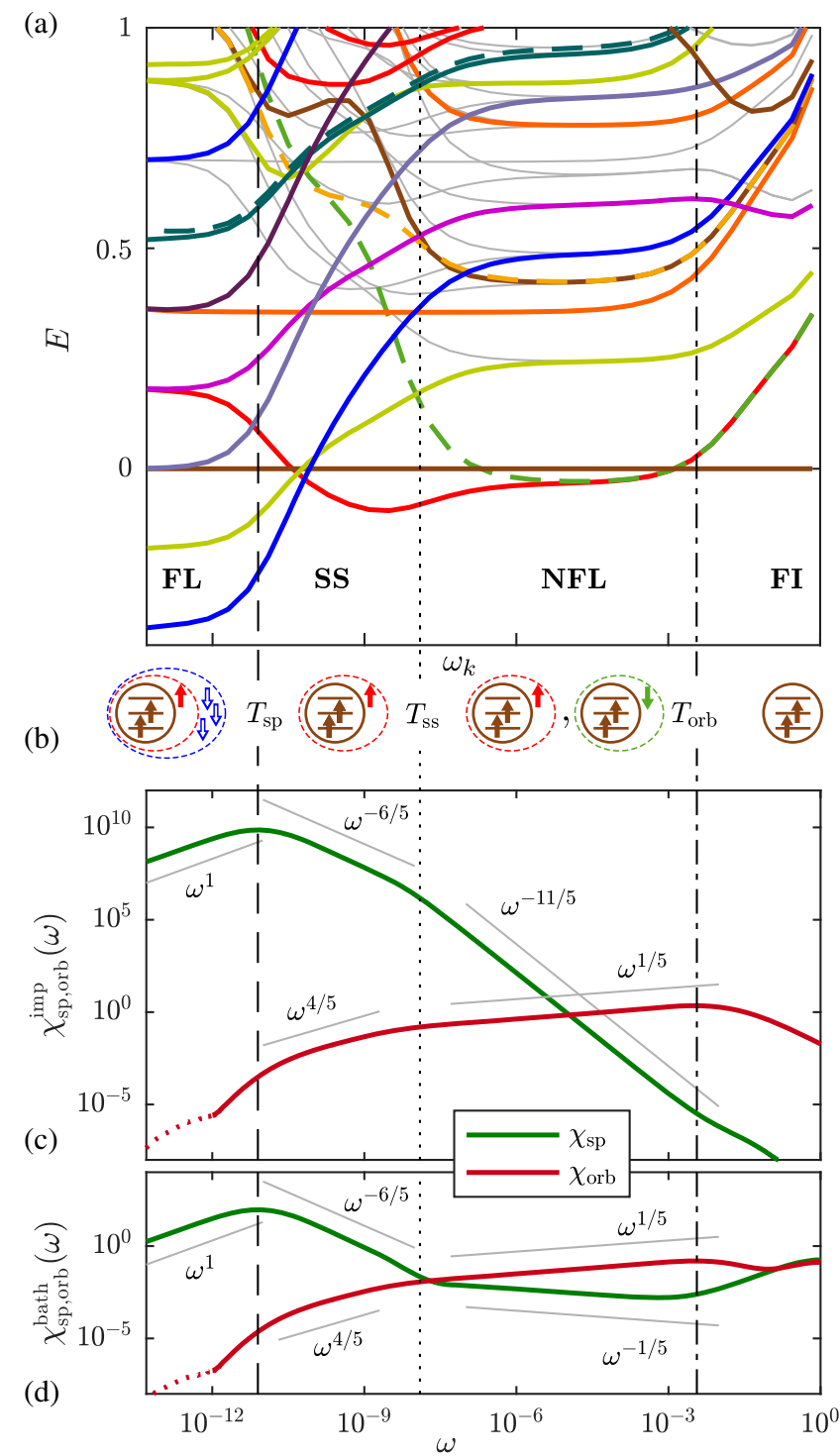

FIG. 2. NRG results for $\mathbf{c}_{0}=\left(J_{0}, K_{0}, I_{0}\right)=\left(10^{-4}, 0.3,0\right)$. (a) Finite-size eigenlevel spectrum computed by $\mathrm{NRG}$, with $\mathcal{E}_{\text {ref }}=\mathcal{E}(0,1, \boxminus)$ as reference energy. Quantum numbers $Q=$ $(q, S, \lambda)$ are shown at the top, and $\rightarrow$ indicates boundary operators obtained via double fusion. (NRG parameters: $\Lambda=2.5$; number of kept multiplets, $N_{\text {keep }}=3000$; half-bandwidth of the bath, $D=1$.) (b) Illustrations of the ground states encountered during the flow. (c),(d) Imaginary part of the spin and orbital susceptibilities of (c) the impurity and (d) the bath site coupled to it (Wilson chain site $k=0$ ). Gray lines show power laws predicted by CFT. Vertical lines show the crossover scales for orbital and spin screening, $T_{\text {orb }}$ and $T_{\mathrm{sp}}$, marking the maxima of $\chi_{\text {orb }}^{\mathrm{imp}}$ and $\chi_{\mathrm{sp}}^{\mathrm{imp}}$, and for spin splitting $T_{\mathrm{ss}}$, marking kinks in $\chi_{\mathrm{sp}, \text { orb }}^{\text {imp,bath }}$. 
To analyze the NFL regime in detail, we choose $I_{0}=0$ and $J_{0} \ll K_{0}$, so that the SOS window becomes very large, with $T_{\text {sp }} \lll T_{\text {orb}}$. Figure 2(a) shows the NRG eigenlevel flow diagram for $\mathbf{c}_{0}=\left(10^{-4}, 0.3,0\right)$. We discern four distinct regimes, separated by three scales, $T_{\mathrm{sp}}, T_{\mathrm{ss}}, T_{\text {orb }}$.

(i) The free-impurity (FI) regime, $\omega_{k}>T_{\text {orb }}$, involves an unscreened impurity, with ground state multiplet $Q=(0,1, \boxminus)$ (flat brown line).

(ii) In the NFL regime, $T_{\mathrm{ss}}<\omega_{k}<T_{\text {orb }}$, two degenerate multiplets, $\left(1, \frac{1}{2}, \bullet\right)$ and $\left(1, \frac{3}{2}, \bullet\right)$ (dashed green and red lines) become the new ground state multiplets. Below the scale $T_{\text {orb }}$, the impurity orbital isopin is thus screened into an orbital singlet $\bullet$ by binding one bath electron, which couples to the impurity spin 1 to yield a total spin of $\frac{1}{2}$ or $\frac{3}{2}$.

(iii) In the spin-splitting (SS) regime, $T_{\mathrm{sp}}<\omega_{k}<T_{\mathrm{ss}}$, the effects of nonzero $J_{0}$ become noticeable, splitting apart $\left(1, \frac{1}{2}, \bullet\right)$ and $\left(1, \frac{3}{2}, \bullet\right)$, the latter drifting down.

(iv) In the FL regime, $\omega_{k}<T_{\mathrm{sp}},(-2,0, \bullet)$ becomes the new ground state multiplet. Below the scale $T_{\mathrm{sp}}$, the spin $3 / 2$ is thus screened into a spin singlet by binding three bath holes, yielding a fully screened impurity. Note the equidistant level spacing, characteristic of a FL.

To further elucidate the consequences of orbital and spin screening, we computed the impurity's zero-temperature orbital and spin susceptibilities,

$$
\begin{aligned}
& \chi_{\mathrm{orb}}^{\operatorname{imp}}(\omega)=-\frac{1}{8 \pi} \sum_{a} \operatorname{Im}\left\langle T^{a} \| T^{a}\right\rangle_{\omega}, \\
& \chi_{\mathrm{sp}}^{\operatorname{imp}}(\omega)=-\frac{1}{3 \pi} \sum_{\alpha} \operatorname{Im}\left\langle S^{\alpha} \| S^{\alpha}\right\rangle_{\omega},
\end{aligned}
$$

where $\langle X \| X\rangle_{\omega}$ refers to the Fourier-transformed retarded correlation functions $-i \Theta(t)\langle[X(t), X(0)]\rangle$ with frequency $\omega$, and analogous susceptibilities, $\chi_{\mathrm{orb}}^{\text {bath }}, \chi_{\mathrm{sp}}^{\text {bath }}$ (involving $\mathbf{J}_{\text {orb }}, \mathbf{J}_{\mathrm{sp}}$ ) for the bath site coupled to it. To this end we used full-density-matrix (FDM) NRG [52] and adaptive broadening of the discrete NRG data [53].

Figures 2(c) and 2(d) show these susceptibilities on a $\log -\log$ scale. $\chi_{\text {orb }}^{\text {imp }}$ and $\chi_{\mathrm{sp}}^{\text {imp }}$ each exhibit a maximum, at two widely different scales, $T_{\text {orb }}$ and $T_{\mathrm{sp}}$, coinciding with the onset of the stationary NFL or FL regimes in Fig. 2(a), respectively. Moreover, the four susceptibilities $\chi_{\text {orb,sp }}^{\mathrm{imp} \text {, bath }}$ all exhibit kinks at a coinciding energy scale $T_{\text {ss }}$, matching the onset of the SS regime in Fig. 2(a). If $\omega$ lies within one of the regimes NFL, SS, or FL, the susceptibilities all show behavior consistent with power laws (gray lines). These power laws can all be explained by CFT, as discussed in Sec. IV. Here we focus on their qualitative features, which by themselves give striking clues about the nature of orbital and spin screening.
In the NFL regime, where $\chi_{\text {orb }}^{\text {imp }}$ decreases with decreasing $\omega$, it exhibits the same power law as $\chi_{\mathrm{orb}}^{\text {bath }}$. In this sense, the impurity's orbital isospin has taken on the same character as that of the bath site it couples to, indicative of orbital screening - in the parlance of AL's CFT analysis, it has been "absorbed" by the bath. This power law $\omega^{1 / 5}$ is nontrivial, differing from the $\omega^{1}$ expected for a fully screened local degree of freedom. This indicates that the local orbital degree of freedom, even while being screened, is still somehow affected by the spin sector. The converse is also true: the onset of orbital screening at $T_{\text {orb }}$ is accompanied by a change in behavior for both spin susceptibilities, $\chi_{\mathrm{sp}}^{\mathrm{imp}}$ and $\chi_{\mathrm{sp}}^{\text {bath }}$. Both increase with decreasing $\omega$, with different powers, indicative of the absence of spin screening in the NFL regime. The exponent for the impurity spin susceptibility, $\chi_{\mathrm{sp}}^{\mathrm{imp}} \sim \omega^{-11 / 5}$, is remarkably large in magnitude. (For comparison, for the standard spin- $1 / 2$, singlechannel Kondo model, $\chi_{\mathrm{sp}}^{\mathrm{imp}} \sim \omega^{-1}$ for $\omega \gtrsim T_{\mathrm{sp}}$.) The highly singular $\omega^{-11 / 5}$ behavior - our perhaps most unexpected result-indicates that the strength of spin fluctuations is strongly amplified by the onset of orbital screening. Our CFT analysis below will reveal the reason for this: orbital screening is accompanied by a renormalization of the local bath spin density at the impurity site.

Upon entering the SS regime, all susceptibility lines show a kink, i.e., change in power law, such that the impurity and bath exponents match not only in the orbital sector, $\chi_{\text {orb }}^{\text {imp }} \sim \chi_{\text {orb }}^{\text {bath }}$, but now also in the spin sector, $\chi_{\mathrm{sp}}^{\text {imp }} \sim \chi_{\mathrm{sp}}^{\text {bath }}$. The latter fact indicates clearly that bath and impurity spin degrees of freedom have begun to interact with each other. However, this is only a precursor to spin screening, since the spin susceptibilities still increase with decreasing $\omega$, albeit with a smaller exponent, $\chi_{\mathrm{sp}}^{\text {imp,bath }} \sim \omega^{-6 / 5}$, than in the NFL regime. However, since the exponent $\gamma=6 / 5$ is larger than 1 , spin fluctuations are anomalously large also in this regime. Importantly, this regime persists also for parameters corresponding to the more realistic $3 \mathrm{oAH}$ model. Indeed, previous DMFT studies for a self-consistent $3 \mathrm{oAH}$ model have yielded behavior for $\chi_{\mathrm{sp}}^{\mathrm{imp}}$ which in the SOS regime is consistent with an exponent of $\gamma=6 / 5$, as further discussed in Secs. IV and VI. Moreover, as mentioned in the Introduction, anomalously large spin fluctuations are of direct relevance for the superconducting state of the iron pnictide Hund metals: in Ref. [14], strong spin fluctuations with $\gamma>1$ were a key ingredient for a proposed explanation for the anomalously large ratio of $2 \Delta_{\max } / T_{c}$ observed experimentally.

Full spin screening eventually sets in in the FL regime, where the spin susceptibilities $\chi_{\mathrm{sp}}^{\text {imp,bath }}$ show the $\omega^{1}$ behavior characteristic of a FL. We expect this behavior also for the orbital susceptibilities, but have not been able to observe it directly, since our results for $\chi_{\text {orb }}^{\text {impath }}$ become numerically unstable when dropping below $\simeq 10^{-5}$ [as indicated by dotted lines in Figs. 2(c) and 2(d)]. 
In the following two sections we explain how the above NRG results can be understood using CFT arguments.

\section{CFT ANALYSIS: SYNOPSIS}

This section presents a synopsis of our CFT analysis. It aims to be accessible also to readers without in-depth knowledge of AL's CFT work on Kondo models. We begin by summarizing AL's strategy for analyzing strong-coupling fixed points of quantum impurity models (Sec. IVA). We then apply it to the NFL fixed point (Sec. IV B) and the FL fixed point (Sec. IV C). A more elaborate discussion of CFT details follows in Sec. V.

\section{A. General strategy}

AL's strategy for determining spectra and correlation functions from CFT involves three key concepts:

(C1) Independent excitations. - The starting assumption is that the low-energy spectrum of a multiorbital Kondo Hamiltonian at a conformally invariant fixed point can be constructed from combinations of independent charge, spin, and orbital excitations. The excitation energies in each sector follow from the commutation relations of certain charge, spin, and orbital operators (these form a so-called Kac-Moody algebra); this is expressed in Eqs. (4) and (12).

(C2) Gluing conditions and fusion rules.-The spectrum of excitations in each sector (charge, spin, orbital) is the same at the free and strong-coupling fixed points. However, the way in which these three types of excitations should be combined to obtain valid many-body excitations, specified by so-called gluing conditions, differs for the free and strong-coupling fixed points. At the former, excitations are glued together in such a manner that a free-fermion spectrum is recovered. At the latter, the impurity has been absorbed by the bath, implying changes in the gluing conditions relative to those of the free fixed point. These changes are governed by so-called fusion rules, which specify how the impurity degrees of freedom should be "added" to those of the bath. This is conceptually similar to angular momentum addition, but with additional constraints to respect the Pauli principle.

(C3) Scaling dimensions.-Once the fusion rules and thus the spectrum of valid many-body excitations is known, the conformal scaling dimensions of operators living at the impurity site can be determined by using the same fusion rules once more ("double fusion"). Because of conformal invariance, the functional form of correlation functions is fully determined by the scaling dimensions of their operators.

In practice, analyzing a conformally invariant strongcoupling fixed point thus consists of three steps: (C1) determine the independent excitations, (C2) use "single fusion" to obtain the strong-coupling gluing conditions, and (C3) use "double fusion" to obtain the scaling dimensions of operators living at the impurity site. Even though AL's justification of this strategy involved sophisticated CFT arguments, its application to an actual model is rather straightforward, once one has determined the appropriate fusion rules. For the $3 \mathrm{soK}$ model, we present tables with the explicit fusion rules in the Supplemental Material (SM) [54], and Table II shows details on the fusion procedure. These tables are also meant to serve as a guide for future applications of AL's methodology.

\section{B. NFL regime}

In the following, we follow this strategy for the NFL fixed point of the 3 soK model.

(C1) The 3 soK model, being spherically symmetric around the origin, describes an effectively one-dimensional system. In the imaginary-time formalism, the field describing the conduction band, $\psi(\tau+i r)$, lives on the upper half of the complex plane, with time $\tau$ on the real and the distance $r$ from the impurity on the imaginary axis. The impurity at $r=0$ constitutes a "boundary" at the real axis. The fixed points of the model, assumed to be scale invariant, can thus be described using $(1+1)$-dimensional boundary CFT.

The bath of the 3 soK model trivially has $\mathrm{U}(1) \times$ $\mathrm{SU}(2) \times \mathrm{SU}(3)$ symmetry. Moreover, since we assumed a flat band, i.e., a linear dispersion, it also has conformal symmetry. The combination of both leads to the symmetry $\mathrm{U}(1) \times \mathrm{SU}(2)_{3} \times \mathrm{SU}(3)_{2}$, where $\mathrm{SU}(2)_{3}$ and $\mathrm{SU}(3)_{2}$ refer to generalizations of the familiar $\mathrm{SU}(2)$ and $\mathrm{SU}(3)$ algebras, known as Kac-Moody algebras $[44,57,58]$. The subscript on $\mathrm{SU}(2)_{3}$ states that only those spin representations are allowed which can be constructed from electrons living on 3 orbitals. In particular, spins larger than $3 / 2$ do not occur in this algebra. The subscript on $\mathrm{SU}(3)_{2}$ indicates analogous restrictions for the allowed SU(3) representations. (The consequences of these restrictions are made explicit in Tables S3 and S2 of the SM [54].)

According to AL [42-46], the fixed points can be analyzed as follows. First, standard $\mathrm{U}(1) \times \mathrm{SU}(2)_{3} \times$ $\mathrm{SU}(3)_{2}$ non-Abelian bosonization is used to decompose the bath Hamiltonian into charge, spin, and orbital contributions,

$$
H_{\mathrm{bath}} \sim \int d r\left[\frac{1}{12} J_{\mathrm{ch}}^{2}(r)+\frac{1}{5} \mathbf{J}_{\mathrm{sp}}^{2}(r)+\frac{1}{5} \mathbf{J}_{\mathrm{orb}}^{2}(r)\right],
$$

with $J_{\mathrm{ch}}(r)=\psi_{m \sigma}^{\dagger}(r) \psi_{m \sigma}(r)$, etc. (We omitted overall prefactors; for a detailed discussion, see Refs. [44,57].) Since $J_{\mathrm{ch}}, \mathbf{J}_{\mathrm{sp}}, \mathbf{J}_{\text {orb }}$ are generators of the $\mathrm{U}(1), \mathrm{SU}(2)_{3}$, $\mathrm{SU}(3)_{2}$ Kac-Moody algebras, respectively, the eigenstates of $H_{\text {bath }}$ can be organized into multiplets forming irreps of the corresponding symmetry groups, labeled by quantum numbers $Q_{\text {bath }}=(q, S, \lambda)$. If the bath is put in a box of 
TABLE I. Left: Five low-lying free-fermion multiplets ( $|\mathrm{FS}\rangle$ denotes the Fermi sea), with quantum numbers $(q, S, \lambda)$, multiplet dimensions $d$, and energies $E(q, S, \lambda)$. Center: "Single fusion" with an impurity $Q_{\mathrm{imp}}=(0,1, \boxminus)$ leads to multiplets with quantum numbers $\left(q, S^{\prime}, \lambda^{\prime}\right)$, dimensions $d^{\prime}$, eigenenergies $E^{\prime}=E\left(q, S, \lambda^{\prime}\right)$, and excitation energies $\delta E^{\prime}=E^{\prime}-E_{\text {min }}^{\prime}$. Right: "Double fusion," which fuses multiplets from the middle column with an impurity in the conjugate representation $\bar{Q}_{\text {imp }}=(0,1, \square)$ [cf. Sec. V B, details on (C3)], yields the multiplets $\left(q, S^{\prime \prime}, \lambda^{\prime \prime}\right)$. These characterize the CFT boundary operators $\hat{O}$, with scaling dimensions $\Delta=E\left(q, S, \lambda^{\prime \prime}\right)$. $\boldsymbol{\Phi}_{\text {orb }}$ and $\boldsymbol{\Phi}_{\text {sp }}$ are the leading boundary operators in the orbital and spin sectors, respectively. In the spin-splitting regime, their roles are taken by $\tilde{\Psi}_{\text {orb }}$ and $\tilde{\Psi}_{\text {sp }}$, respectively. "Bare" free-fermion versions of these boundary operators, having the same quantum numbers, are listed on the very right. For clarity, not all possible multiplets arising from single and double fusion are shown. A more comprehensive list is given in Table II.

\begin{tabular}{|c|c|c|c|c|c|c|c|c|c|c|c|c|c|c|c|c|}
\hline \multirow[b]{2}{*}{ State } & \multicolumn{5}{|c|}{ Free fermions } & \multicolumn{5}{|c|}{ Single fusion } & \multicolumn{6}{|c|}{ Double fusion } \\
\hline & $q$ & $S$ & $\lambda$ & $d$ & $E$ & $q$ & $S^{\prime}$ & $\lambda^{\prime}$ & $d^{\prime}$ & $\delta E^{\prime}$ & $q$ & $S^{\prime \prime}$ & $\lambda^{\prime \prime}$ & $\Delta$ & $\hat{O}$ & $\hat{O}_{\text {bare }}$ \\
\hline$\overline{\mid F S}\rangle$ & 0 & 0 & $\bullet$ & 1 & 0 & 0 & 1 & 曰 & 9 & $\frac{1}{30}$ & 0 & 0 & $\square$ & $\frac{3}{5}$ & $\boldsymbol{\Phi}_{\text {orb }}$ & $\mathbf{T}, \mathbf{J}_{\text {orb }}$ \\
\hline$\psi_{m \sigma}^{\dagger}|\mathrm{FS}\rangle$ & 1 & $\frac{1}{2}$ & $\square$ & 6 & $\frac{1}{2}$ & 1 & $\left\{\frac{1}{2}, \frac{3}{2}\right\}$ & $\bullet$ & 6 & 0 & & $\cdots$ & & & & \\
\hline$\psi_{m \sigma}|\mathrm{FS}\rangle$ & -1 & $\frac{1}{2}$ & $\boxminus$ & 6 & $\frac{1}{2}$ & -1 & $\frac{1}{2}$ & $\square$ & 6 & $\frac{4}{15}$ & -1 & $\frac{1}{2}$ & $\square$ & $\frac{9}{10}$ & $\tilde{\Psi}_{\text {orb }}$ & $\begin{array}{l}\left(\psi_{l \sigma}^{\dagger} \psi_{l \sigma}-\psi_{m \sigma}^{\dagger} \psi_{m \sigma}\right) \psi_{n \sigma}, \\
\psi_{l \sigma}^{\dagger} \psi_{m \sigma} \psi_{n \sigma}, l \neq m \neq n\end{array}$ \\
\hline $\mathbf{J}_{\text {sp-orb }}|\mathrm{FS}\rangle$ & 0 & 1 & $\square$ & 24 & 1 & 0 & 0 & $\boxminus$ & 3 & $\frac{13}{30}$ & 0 & 1 & - & $\frac{2}{5}$ & $\boldsymbol{\Phi}_{\mathrm{sp}}$ & $\mathbf{J}_{\mathrm{sp}}$ \\
\hline$\cdots$ & -3 & $\frac{1}{2}$ & $\square$ & 16 & $\frac{3}{2}$ & -3 & $\frac{1}{2}$ & $\boxminus$ & 6 & $\frac{14}{15}$ & -3 & $\frac{1}{2}$ & $\bullet$ & $\frac{9}{10}$ & $\tilde{\Psi}_{\mathrm{sp}}$ & $\psi_{1 \sigma} \psi_{2 \sigma} \psi_{3 \bar{\sigma}}$ \\
\hline
\end{tabular}

finite size, the corresponding free-fermion excitation eigenenergies $E(q, S, \lambda)$ are discrete and simple functions of the quantum numbers [see Eq. (12)].

(C2) Next, we include the interaction with the impurity in the orbital sector $\left(K_{0}>0, J_{0}=I_{0}=0\right)$ to describe the properties of the NFL fixed point $\mathbf{c}_{\mathrm{NFL}}^{*}$. The bosonized $H_{\text {bath }}$ is quadratic in $\mathbf{J}_{\text {orb }}$, whereas the coupling term $H_{\text {int }}=$ $K_{0} \mathbf{T} \cdot \mathbf{J}_{\text {orb }}(r=0)$ is linear. The latter can thus be absorbed into the former, in the spirit of "completing the square." $\mathrm{AL}$ conjectured that at the strong-coupling fixed point, this replacement takes the form

$$
\mathbf{J}_{\text {orb }}(r) \mapsto \mathcal{J}_{\text {orb }}(r)=\mathbf{J}_{\text {orb }}(r)+\delta(r) \mathbf{T},
$$

with $\mathcal{J}_{\text {orb }}$ satisfying the same Kac-Moody algebra as $\mathbf{J}_{\text {orb }}$. At the strong-coupling fixed point, the Hamiltonian can thus be expressed as $H=H_{\text {bath }}\left[\mathbf{J}_{\text {orb }}\right]+H_{\text {int }}=H_{\text {bath }}\left[\mathcal{J}_{\text {orb }}\right]$ (more details can be found in Sec. V B and Ref. [43]).

It follows immediately that at the fixed point, the spectrum of irreps of the full Hamiltonian can be obtained by combining the irreps of bath and impurity degrees of freedom, $Q_{\text {bath }} \otimes Q_{\text {imp }}=\sum_{\oplus} Q^{\prime}$, and using "fusion rules" to deduce the resulting irreps $Q^{\prime}$. This is conceptually similar to coupling two $\mathrm{SU}(2)$ spins, $\mathbf{S}^{\prime \prime}=\mathbf{S}+\mathbf{S}^{\prime}$, decomposing the direct product of their irreps as $S \otimes S^{\prime}=$ $\sum_{\oplus} S^{\prime \prime}$, and deducing that $S^{\prime \prime}$ ranges from $\left|S-S^{\prime}\right|$ to $S+S^{\prime}$. However, in the present context, specific assumptions must be made about which degrees of freedom are involved in the screening processes and which are not, and for those which are, Kac-Moody fusion rules have to be used when combining irreps. For the present situation, we have $Q_{\text {bath }}=(q, S, \lambda)$ and $Q_{\text {imp }}=(0,1, \boxminus)$, and place ourselves $a t$ the NFL fixed point, where bath and impurity couple only in the orbital sector.
To find the allowed irreps $Q^{\prime}=\left(q^{\prime}, S^{\prime}, \lambda^{\prime}\right)$, we therefore posit the following fusion strategy (inspired by and generalizing that of AL [42-46]). In the charge sector, $q_{\text {imp }}=0$ trivially implies that $q^{\prime}=q$. In the orbital sector, the impurity's orbital isospin is coupled to that of the bath [Eq. (1)] and absorbed by it according to Eq. (5); hence, $\lambda \otimes \lambda_{\text {imp }}=\sum_{\oplus} \lambda^{\prime}$ is governed by the fusion rules of the $\mathrm{SU}(3)_{2}$ Kac-Moody algebra. By contrast, in the spin sector the impurity spin is a spectator, decoupled from the bath (we are at $\mathbf{c}_{\mathrm{NFL}}^{*}$, where $J_{0}=I_{0}=0$ ); hence, $S \otimes S_{\mathrm{imp}}=$ $\sum_{\oplus} S^{\prime}$ is governed by the fusion rules of the $\mathrm{SU}(2)$ Lie algebra [not the $\mathrm{SU}(2)_{3}$ Kac-Moody algebra]. The set of excitations $\left(q, S^{\prime}, \lambda^{\prime}\right)$ so obtained have energies given by $E\left(q, S, \lambda^{\prime}\right)$, not $E\left(q, S^{\prime}, \lambda^{\prime}\right)$, since $H_{\text {int }}$ only acts in the orbital sector. A more complete discussion of our "fusion hypothesis" is given in Sec. V B. The resulting spectrum reproduces the NRG spectrum in the NFL fixed point regime (see Table II).

Table I exemplifies a few many-body states obtained via this fusion scheme (AL called it single fusion, in distinction from a second fusion step, discussed below). In particular, the degenerate ground state multiplets of $\mathbf{c}_{\mathrm{NFL}}^{*},\left(1, \frac{1}{2}, \bullet\right)$ and $\left(1, \frac{3}{2}, \bullet\right)$ [cf. Fig. 2(a)], arise via fusion of a one-particle bath excitation, $\left(+1, \frac{1}{2}, \square\right)$, with the impurity $(0,1, \square)$, schematically depicted in Fig. 2(b).

(C3) Next, we want to compute the leading scaling behavior of spin and orbital correlation functions at the impurity site, i.e., on the boundary of the CFT. The absorption of the impurity into the bath (bulk) Hamiltonian translates, in CFT language, to a change in the boundary condition imposed on the theory at $r=0$. As a result, a new set of "boundary operators," i.e., local operators living at the impurity site, appear in the theory. 
These fully characterize the strong-coupling fixed point. Each boundary operator can be viewed as the renormalized version, resulting from the screening process, of some bare local operator having the same quantum numbers.

According to AL, the boundary operators can be obtained via a second fusion step (double fusion) (cf. Refs. [44-46] and Appendix C of Ref. [57]). Each multiplet $\left(q, S^{\prime \prime}, \lambda^{\prime \prime}\right)$ resulting from double fusion is associated with a boundary operator $\hat{O}$ with the same quantum numbers, and a scaling dimension given by $\Delta=E\left(q, S, \lambda^{\prime \prime}\right)$ (cf. Table I). The realization that the scaling dimensions of boundary operators are related to finite-size excitation energies is due to Cardy [59]. Using a conformal mapping, he mapped the complex upper half-plane to a strip of infinite length and finite width, in such a way that the nontrivial boundary condition of the half-plane is mapped to both boundaries of the strip. He then showed that the boundary operators of the half-plane and their scaling dimensions can be associated with the finite-size spectrum of a Hamiltonian defined along the width of this strip. Since the strip has two nontrivial boundaries, one on each side, the finite-size spectrum can be found using a double-fusion procedure. The scaling dimensions of the boundary operators fully determine their time- or frequency-dependent correlators, $\langle\hat{O}(t) \hat{O}(0)\rangle \sim t^{-2 \Delta}$ and $\langle\hat{O} \| \hat{O}\rangle_{\omega} \simeq \omega^{2 \Delta-1}$.

To explain the power laws found in the NFL regime of Figs. 2(c) and 2(d), and particularly the fact that there $\chi_{\text {orb }}^{\text {imp }}$

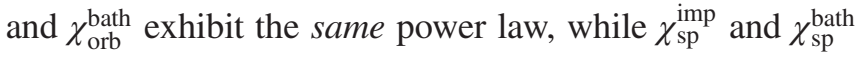
do not, we posit that the local operators in the orbital and spin exchange terms of Eq. (1) are renormalized to

$$
\mathbf{J}_{\text {orb }} \mapsto \boldsymbol{\Phi}_{\text {orb }}, \quad \mathbf{T} \mapsto \boldsymbol{\Phi}_{\text {orb }}, \quad \mathbf{J}_{\mathrm{sp}} \mapsto \boldsymbol{\Phi}_{\mathrm{sp}}, \quad \mathbf{S} \mapsto \mathbf{S} .
$$

Here $\boldsymbol{\Phi}_{\text {orb }}$ has quantum numbers $(0,0, \square)$ (same as $\mathbf{T}, \mathbf{J}_{\text {orb }}$ ) and dimension $\Delta_{\text {orb }}=\frac{3}{5}$, while $\boldsymbol{\Phi}_{\mathrm{sp}}$ has quantum numbers $(0,1, \bullet)\left(\right.$ same as $\left.\mathbf{S}, \mathbf{J}_{\mathrm{sp}}\right)$ and $\Delta_{\mathrm{sp}}=\frac{2}{5}$ (cf. Table I). The local impurity and bath orbital susceptibilities thus both scale as

$$
\chi_{\text {orb }}^{\text {imp,bath }} \sim\left\langle\boldsymbol{\Phi}_{\text {orb }}|| \boldsymbol{\Phi}_{\text {orb }}\right\rangle_{\omega} \sim \omega^{2 \Delta_{\text {orb }}-1}=\omega^{1 / 5},
$$

and the bath spin susceptibility as

$$
\chi_{\mathrm{sp}}^{\text {bath }} \sim\left\langle\boldsymbol{\Phi}_{\mathrm{sp}}|| \boldsymbol{\Phi}_{\mathrm{sp}}\right\rangle_{\omega} \sim \omega^{2 \Delta_{\mathrm{sp}}-1}=\omega^{-1 / 5} .
$$

By contrast, the impurity spin $\mathbf{S}$ is not renormalized, because at the fixed point $\mathbf{c}_{\mathrm{NFL}}^{*}$, where $J_{0}=0$, it is decoupled from the bath. Thus its scaling dimension is zero. The leading behavior of $\chi_{\mathrm{sp}}^{\mathrm{imp}}$ is obtained by now taking $J_{0} \neq 0$ but very small $\left(\ll K_{0}\right)$, and doing secondorder perturbation theory in the renormalized spin exchange interaction. Thus, $\chi_{\mathrm{sp}}^{\mathrm{imp}}$ is proportional to the Fourier transform of $\left\langle\mathbf{S}(t) \mathbf{S}(0)\left(\int d t^{\prime} J_{0} \mathbf{S} \cdot \mathbf{\Phi}_{\mathrm{sp}}\right)^{2}\right\rangle$, and power counting yields

$$
\chi_{\mathrm{sp}}^{\mathrm{imp}} \sim \omega^{2 \Delta_{\mathrm{sp}}-3}=\omega^{-11 / 5} .
$$

The above predictions are all borne out in Figs. 2(c) and $2(\mathrm{~d})$.

The remarkably large negative exponent, $-\frac{11}{5}$, for $\chi_{\mathrm{sp}}^{\mathrm{imp}}$ reflects the fact that the renormalized spin exchange interaction $J_{0} \mathbf{S} \cdot \boldsymbol{\Phi}_{\mathrm{sp}}$, with scaling dimension $\frac{2}{5}<1$, is a relevant perturbation. Its strength, though initially miniscule if $J_{0} \ll 1$, grows under the RG flow, causing a crossover away from $\mathbf{c}_{\mathrm{NFL}}^{*}$ for $\omega \lesssim T_{\mathrm{ss}}$. This is reflected in the level crossings around $T_{\mathrm{ss}}$ in the NRG eigenlevel flow of Fig. 2. In particular, the double-fusion parent multiplets for $\boldsymbol{\Phi}_{\text {orb }}$ and $\boldsymbol{\Phi}_{\text {sp }}$, namely $(0,1, \boxminus)$ and $(0,0, \boxminus)$, undergo level crossings with the downwardmoving multiplets $\left(-1, \frac{1}{2}, \square\right)$ and $\left(-3, \frac{1}{2}, \boxminus\right)$, respectively. These in turn are double-fusion parent multiplets for the boundary operators $\tilde{\Psi}_{\text {orb }}$ and $\tilde{\Psi}_{\text {sp }}$, with scaling dimensions $\tilde{\Delta}_{\text {orb }}=\tilde{\Delta}_{\text {sp }}=\frac{9}{10}$ (Table I). To explain the SS regime of Figs. 2(c) and 2(d), and particularly that there the power laws for $\chi^{\mathrm{imp}}$ and $\chi^{\text {bath }}$ match in both the orbital and spin sectors, we posit the RG replacements

$$
\begin{aligned}
\mathbf{J}_{\mathrm{orb}} & \mapsto \tilde{\Psi}_{\mathrm{orb}}, \quad \mathbf{T} \mapsto \tilde{\Psi}_{\mathrm{orb}}, \\
\mathbf{J}_{\mathrm{sp}} & \mapsto \mathbf{S}+\tilde{\Psi}_{\mathrm{sp}}, \quad \mathbf{S} \mapsto \mathbf{S}+\tilde{\Psi}_{\mathrm{sp}} .
\end{aligned}
$$

Here $\mathbf{S}+\tilde{\Psi}_{\mathrm{sp}}$ is symbolic notation for some linear admixture of both operators, induced by the action of the renormalized spin exchange interaction. We thus obtain

$$
\chi_{\text {orb }}^{\text {impath }} \sim\left\langle\tilde{\Psi}_{\text {orb }}|| \tilde{\Psi}_{\text {orb }}\right\rangle_{\omega} \sim \omega^{2 \tilde{\Delta}_{\text {orb }}-1}=\omega^{4 / 5},
$$

and the leading contribution to $\chi_{\mathrm{sp}}^{\mathrm{imp}}$ and $\chi_{\mathrm{sp}}^{\text {bath }}$, obtained by perturbing $\langle\mathbf{S}(t) \mathbf{S}(0)\rangle$ to second order in $\mathbf{S} \tilde{\Psi}_{\text {sp }}$ [60], is

$$
\chi_{\mathrm{sp}}^{\mathrm{imp}, \text { bath }} \sim \omega^{2 \tilde{\Delta}_{\mathrm{sp}}-3}=\omega^{-6 / 5} .
$$

This reproduces the power laws found in Figs. 2(c) and 2(d).

Remarkably, $\chi_{\mathrm{sp}}^{\mathrm{imp}} \sim \omega^{-6 / 5}$ behavior has also been found in studies of the self-consistent 3oAH model arising in our DMFT investigations of the three-orbital Hubbard-Hund model for Hund metals. For the 3oAH model the spinorbital coupling $I_{0}$ in Eq. (1) is always nonzero, so that a fully fledged NFL does not emerge-instead, $T_{\text {orb }}$ and $T_{\text {ss }}$ effectively coincide (as further discussed in Sec. VI). However, the SS regime between $T_{\text {sp }}$ and $T_{\text {ss }} \simeq T_{\text {orb }}$ can be quite wide, typically at least an order of magnitude. In Fig. 3(c) of Ref. [22], the behavior of $\chi_{\mathrm{sp}}^{\mathrm{imp}}$ in this regime (between the vertical solid and black lines there) is consistent with $\omega^{-6 / 5}$ behavior. Though this fact was not noted in Ref. [22], it was subsequently pointed out in Ref. [14] (see Fig. S1 of their Supplemental Material). 
Behavior consistent with $\chi_{\mathrm{sp}}^{\mathrm{imp}} \sim \omega^{-6 / 5}$ can also be seen in Figs. 5.1(c) and 5.1(d) of Ref. [47], as discussed on p. 152 therein. The explanation for this behavior presented here, via a CFT analysis of the NFL and SS regimes, is one of the main results of this work, and the justification for the first part of the title of this paper.

\section{Fermi-liquid regime}

As mentioned above, the low-energy regime below $T_{\mathrm{sp}}$ is a FL. The fixed-point spectrum at $\mathbf{c}_{\mathrm{FL}}^{*}$ can be obtained by fusing a free-fermion spectrum with an impurity with $Q_{\text {imp }}=\left(1, \frac{3}{2}, \bullet\right)$, representing the effective local degree of freedom obtained after completion of orbital screening (see Table III). Since the ground state describes a fully screened orbital and spin singlet, it actually is the singlet of a larger symmetry group, $\mathrm{U}(1) \times \mathrm{SU}(6)$. Indeed, the fixedpoint spectrum at $\mathbf{c}_{\mathrm{FL}}^{*}$ matches that of the $\mathrm{U}(1) \times \mathrm{SU}(6)$ symmetric Kondo model. We demonstrate this, using both NRG and CFT with $\mathrm{SU}(6)_{1}$ fusion rules, in Sec. V E (see Table IV). The FL nature of the ground state is also borne out by the $\omega^{1}$ scaling of $\chi_{\mathrm{sp}}^{\text {imp, bath }}$ in the FL regime of Figs. 2(c) and 2(d).

\section{CFT ANALYSIS: DETAILS}

We now provide technical details for our CFT analysis of the NFL and FL fixed points of the three-orbital Kondo model discussed in Secs. III and IV. We closely follow the strategy devised by Affleck and Ludwig for their pioneering treatment of the strong-coupling fixed points of Kondo models [42-46] (for pedagogical reviews, see Refs. [58,61] and Appendixes A-D of Ref. [57]). In a series of works, they considered a variety of Kondo models of increasing complexity. These include the standard one-channel, SU(2) spin Kondo model with a spin exchange interaction between bath and impurity with $\mathrm{U}(1) \times \mathrm{SU}(2)_{1}$ symmetry; a spinful $k$-channel bath coupled to an $\mathrm{SU}(2)$ impurity $\left[\mathrm{U}(1) \times \mathrm{SU}(2)_{k} \times \mathrm{SU}(\mathrm{k})_{2}\right.$ symmetry], and a $\mathrm{SU}(N) k$-channel bath coupled to an $\mathrm{SU}(N)$ impurity $\left[\mathrm{U}(1) \times \mathrm{SU}(\mathrm{N})_{k} \times \mathrm{SU}(\mathrm{k})_{N}\right.$ symmetry].

Our 3 soK model features a spinful three-channel bath and an $\mathrm{SU}(2)_{\mathrm{sp}} \times \mathrm{SU}(3)_{\text {orb }}$ impurity $\left[\mathrm{U}(1) \times \mathrm{SU}(2)_{3} \times\right.$ $\mathrm{SU}(3)_{2}$ symmetry]. The impurity multiplet is a direct product of a spin triplet $(S=1)$ and an orbital triplet $(\lambda=\boxminus)$. Its direct-product structure is more general than any of the cases considered by AL. [A two-channel version of our model, with $\mathrm{U}(1) \times \mathrm{SU}(2)_{2} \times \mathrm{SU}(2)_{2}$ symmetry, has been studied by Ye [48], which we discuss in the Appendix.] However, at the NFL fixed point $\mathbf{c}_{\mathrm{NFL}}^{*}$ of our model, where $J_{0}=I_{0}=0$, the impurity's $\mathrm{SU}(2)$ spin is a decoupled, threefold degenerate spectator degree of freedom. Hence AL's analysis [46] can be employed, with $N=3$ and $k=2$ channels, modulo some minor changes to account for the impurity spin.
By contrast, in the spin-splitting crossover regime the spin exchange interaction comes to life, so that the impurity's SU(2) spin degrees of freedom cease to be mere spectators. This regime thus lies outside the realm of cases studied by AL; in particular, it is not manifestly governed by the NFL fixed point $\mathbf{c}_{\mathrm{NFL}}^{*}$, or any other well-defined fixed point. Correspondingly, our discussion of this crossover regime in Sec. V C 2 is more speculative than that of the NFL regime, though our heuristic arguments are guided by and consistent with our NRG results.

Finally, for our model's FL fixed point $\mathbf{c}_{\mathrm{FL}}^{*}$, we are again in well-chartered territory: it can be understood by applying AL's strategy to an SU(6) one-channel bath coupled to an $\mathrm{SU}(6)$ impurity $\left[\mathrm{U}(1) \times \mathrm{SU}(6){ }_{1}\right.$ symmetry].

Below we assume the reader to be familiar with AL's work and just focus on documenting the details of our analysis. Section VA describes how the free-fermion bath spectrum is decomposed into charge, spin, and orbital excitations using $\mathrm{U}(1) \times \mathrm{SU}(2)_{3} \times \mathrm{SU}(3)_{2}$ non-Abelian bosonization. Section V B derives the finite-size spectrum and boundary operators of the NFL fixed point via single and double fusion, using the fusion rules of the $\mathrm{SU}(3)_{2}$ Kac-Moody algebra in the orbital sector and the SU(2) Lie algebra in the spin sector. Section V C describes the computation of the spin and orbital susceptibilities in the NFL and SS regimes, linking AL's strategy for computing such quantities to the compact scaling arguments used in Sec. IV. Section V D presents our results for the impurity spectral function in the NFL regime. Finally, Sec. VE, devoted to the FL regime, shows how its spectrum can be derived using either $\mathrm{SU}(2)_{3}$ fusion rules in the spin sector or $\mathrm{SU}(6)_{1}$ fusion rules in the flavor (combined spin + orbital) sector.

\section{A. Non-Abelian $\mathrm{U}(1) \times \mathrm{SU}(2)_{3} \times \mathrm{SU}(3)_{2}$ bosonization}

(C1) The first step of AL's CFT approach for multichannel Kondo models is to use non-Abelian bosonization to decompose the bath degrees of freedom into charge, spin, and orbital excitations in a manner respecting the symmetry of the impurity-bath exchange interactions. Our 3 soK model features a spinful three-channel bath, with $H_{\text {bath }}=\sum_{p m \sigma} \varepsilon_{p} \psi_{p m \sigma}^{\dagger} \psi_{p m \sigma}$. We assume a linear dispersion, $\varepsilon_{p}=\hbar v_{F} p$, with $\hbar v_{F}=1$. Using nonAbelian bosonization with the $\mathrm{U}(1) \times \mathrm{SU}(2)_{3} \times \mathrm{SU}(3)_{2}$ Kac-Moody current algebra, the spectrum of bath excitations can be expressed as (see Refs. [42,44] or Appendix A of Ref. [57])

$$
\begin{gathered}
E(q, S, \lambda)=\frac{1}{12} q^{2}+\frac{1}{5} \kappa_{2}(S)+\frac{1}{5} \kappa_{3}(\lambda)+\ell, \\
\kappa_{2}(S)=S(S+1), \\
\kappa_{3}(\lambda)=\frac{1}{3}\left(\lambda_{1}^{2}+\lambda_{2}^{2}+\lambda_{1} \lambda_{2}+3 \lambda_{1}+3 \lambda_{2}\right) .
\end{gathered}
$$


Here $\kappa_{2}(S)$ and $\kappa_{3}(\lambda)$ are the eigenvalues of the quadratic Casimir operators of the $\mathrm{SU}(2)$ and $\mathrm{SU}(3)$ Lie algebras, respectively [62]. $q \in \mathbb{Z}$ is the $\mathrm{U}(1)$ charge quantum number, $S \in \frac{1}{2} \mathbb{Z}$ the $S U(2)$ spin quantum number, and $\lambda=\left(\lambda_{1}, \lambda_{2}\right)$ the $\mathrm{SU}(3)$ orbital quantum number, denoting a Young diagram with $\lambda_{j} j$-row columns:

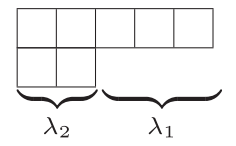

$$
\lambda_{1}=\text { number of 1-row columns }
$$$$
\lambda_{2}=\text { number of 2-row columns }
$$

Finally, $\ell \in \mathbb{Z}$ counts higher-lying "descendent" excitations; for present purposes it suffices to set $\ell=0$.

The free-fermion spectrum of $H_{\text {bath }}$ is recovered from Eq. (12) by imposing free-fermion "gluing conditions," allowing only those combinations of quantum numbers $(q, S, \lambda)$ for which $E(q, S, \lambda)$ is an integer multiple of $1 / 2$. The resulting multiplets are listed in the left-hand column ("Free fermions") of Table II.

\section{B. Non-Fermi-liquid fixed point}

(C2) We now focus on the NFL fixed point of the 3 soK model, at $\mathbf{c}_{\mathrm{NFL}}^{*}$, where $\left(J_{0}, K_{0}, I_{0}\right)=(0,1,0)$. According to AL's general strategy, the orbital isospin $T$ can be then "absorbed" by the bath through the substitution

$$
\mathbf{J}_{\mathrm{orb}, n} \mapsto \mathcal{J}_{\mathrm{orb}, n}=\mathbf{J}_{\mathrm{orb}, n}+\mathbf{T} .
$$

Here $\mathbf{J}_{\mathrm{orb}, n}$ and $\mathcal{J}_{\mathrm{orb}, n}$ are Fourier components ( $n$ being a Fourier index) of the bare and bulk orbital isospin currents, respectively, defined for a bath in a finite-sized box. (The local bath operator $\mathbf{J}_{\text {orb }}$ is proportional to $\sum_{n \in \mathbb{Z}} \mathbf{J}_{\text {orb }, n}$.) The right-hand side of Eq. (13) is reminiscent of the addition of Lie algebra generators, $\mathbf{S}^{\prime}=\mathbf{S}+\tilde{\mathbf{S}}$, when performing a direct product decomposition, $S \otimes \tilde{S}=\sum_{\oplus} S^{\prime}$, of $\mathrm{SU}(2)$ multiplets. The terms added in Eq. (13), however, generate two different algebras: $\mathbf{J}_{\mathrm{orb}, n}$ are generators of the $\mathrm{SU}(3)_{2}$ $\mathrm{KM}$ algebra, $\mathbf{T}$ of the SU(3) Lie algebra. AL proposed a remarkable fusion hypothesis for dealing with such situations (and confirmed its veracity by detailed comparisons to Bethe ansatz and NRG computations). For the present context their fusion hypothesis states: the eigenstates of the combined bath + impurity system can be obtained by combining (or "fusing") their orbital degrees of freedom, $\lambda \otimes \lambda_{\text {imp }}=\sum_{\oplus} \lambda^{\prime}$, using the fusion rules of the $\mathrm{SU}(3)_{2}$ $\mathrm{KM}$ algebra, as though the impurity's orbital multiplet were an $\mathrm{SU}(3)_{2}$, not $\mathrm{SU}(3)$, multiplet. The $\mathrm{SU}(3)_{2}$ fusion rules are depicted in Table S2 of the Supplemental Material [54].

Having discussed orbital fusion, we now turn to the spin sector-how should the impurity's spectator spin be dealt with? This question goes beyond the scope of AL's work, who did not consider impurities with spectator degrees of freedom. We have explored several spin fusion strategies and concluded that the following one yields spectra consistent with NRG: In parallel to orbital fusion, the bath and impurity spin degrees should be combined too, as

TABLE II. Fusion table for orbital screening at the NFL fixed point $\mathbf{c}_{\mathrm{NFL}}^{*}$ of the 3soK model. Left: The 14 lowest low-lying freefermion multiplets $(q, S, \lambda)$, with multiplet dimensions $d$ and energies $E(q, S, \lambda)$, computed using Eqs. (12) and Table S1 of the SM [54]. Center: Single fusion with a $Q_{\mathrm{imp}}=(0,1, \boxminus)$ impurity, using $\mathrm{SU}(2)$ fusion rules in the spin sector and $\mathrm{SU}(3)_{2}$ fusion rules (listed in Table S2 of the SM [54]) in the orbital sector. This yields multiplets $\left(q, S^{\prime}, \lambda^{\prime}\right)$, with dimensions $d^{\prime}$, energies $E^{\prime}=E\left(q, S, \lambda^{\prime}\right)$, and excitation energies $\delta E^{\prime}=E^{\prime}-E_{\min }^{\prime}$. These are compared to the values, $E_{\mathrm{NRG}}$, computed by NRG for $\left(J_{0}, K_{0}, I_{0}\right)=(0,0.3,0)$. The NRG energies have been shifted and rescaled such that the lowest energy is zero and the second-lowest values for $E_{\mathrm{NRG}}$ and $\delta E^{\prime}$ match. The single-fusion and NRG spectra agree well (deviations $\lesssim 10 \%$ ). Right: Double fusion, which fuses multiplets from the middle column with an impurity in the conjugate representation $\bar{Q}_{\text {imp }}=(0,1, \square)$, yields the quantum numbers $\left(q, S^{\prime \prime}, \lambda^{\prime \prime}\right)$. These characterize the CFT

\begin{tabular}{|c|c|c|c|c|c|c|c|c|c|c|c|c|c|c|c|c|}
\hline \multicolumn{5}{|c|}{ Free fermions } & \multicolumn{6}{|c|}{ Single fusion, with $Q_{\mathrm{imp}}=(0,1, \boxminus)$} & \multirow{2}{*}{$\begin{array}{c}\mathrm{NRG} \\
E_{\mathrm{NRG}}\end{array}$} & \multicolumn{5}{|c|}{ Double fusion, with $\bar{Q}_{\mathrm{imp}}=(0,1, \square)$} \\
\hline$q$ & $S$ & $\lambda$ & $d$ & $E$ & $q$ & $S^{\prime}$ & $\lambda^{\prime}$ & $d^{\prime}$ & $E^{\prime}$ & $\delta E^{\prime}$ & & $q$ & $S^{\prime \prime}$ & $\lambda^{\prime \prime}$ & $\Delta$ & $\hat{O}$ \\
\hline 0 & 0 & • & 1 & 0 & 0 & 1 & $\square$ & 9 & $\frac{4}{15}$ & $\frac{1}{30}(0.033)$ & 0.033 & 0 & $\begin{array}{l}1 \\
2\end{array}$ & $\begin{array}{l}\bullet \\
\square \\
\bullet \\
\bullet \\
\square \\
\bullet \\
\bullet \\
\square\end{array}$ & $\begin{array}{l}0 \\
\frac{3}{5} \\
0 \\
\frac{3}{5} \\
0 \\
\frac{3}{5}\end{array}$ & $\begin{array}{c}\mathbb{1} \\
\boldsymbol{\Phi}_{\text {orb }}\end{array}$ \\
\hline
\end{tabular}
boundary operators $\hat{O}$, with scaling dimensions $\Delta=E\left(q, S, \lambda^{\prime \prime}\right)$. 
TABLE II. (Continued)

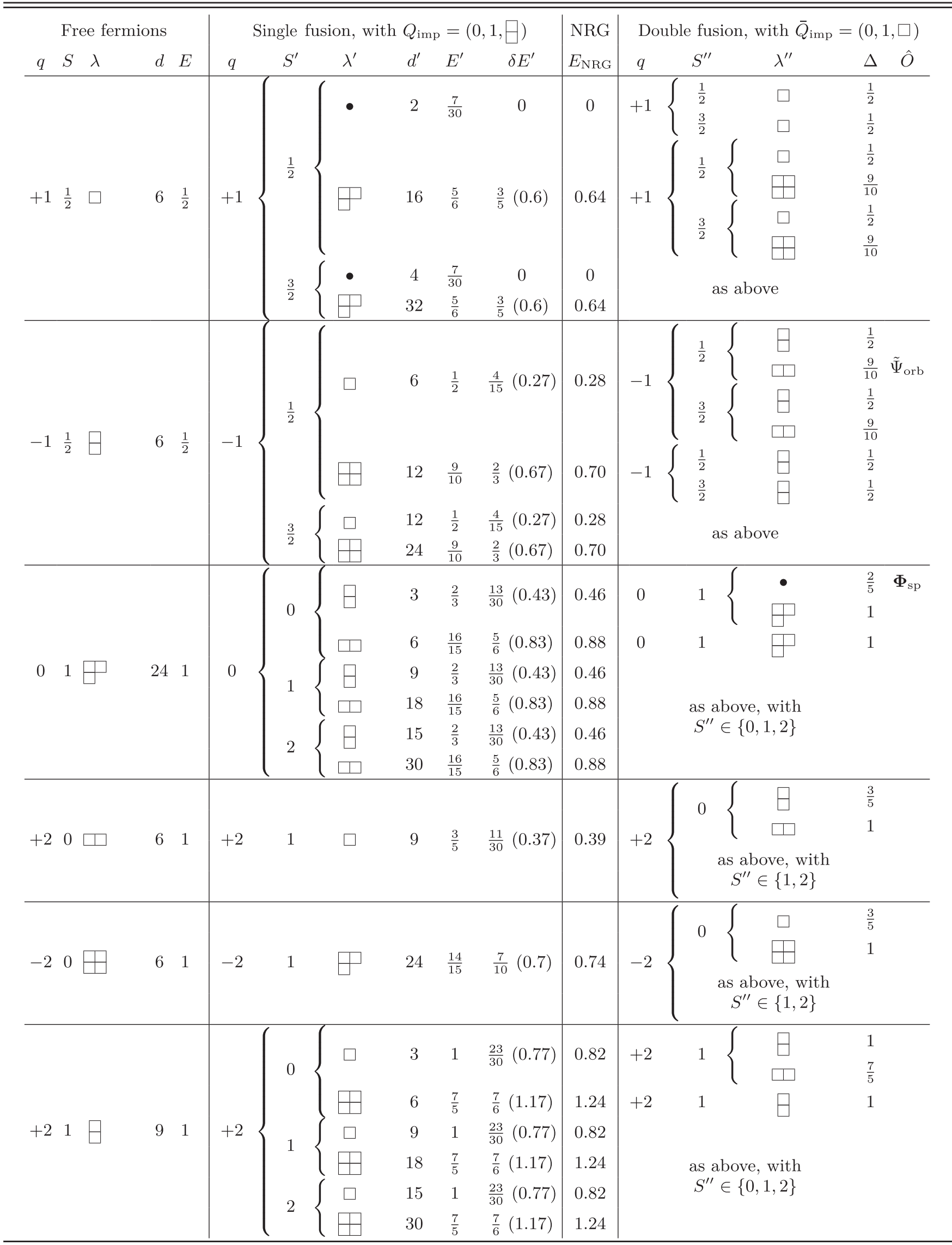


TABLE II. (Continued)

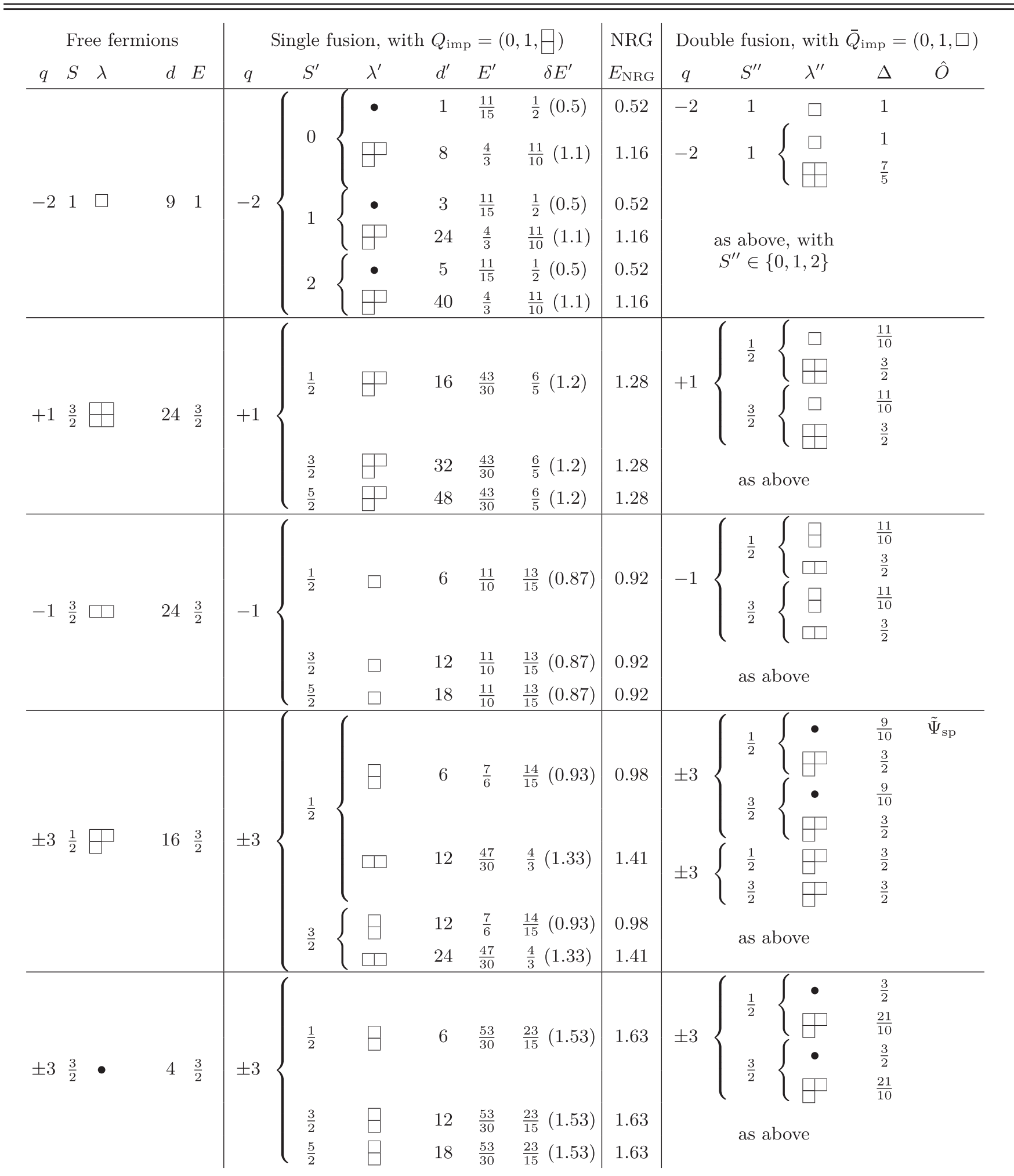

$S \otimes S_{\mathrm{imp}}=\sum_{\oplus} S^{\prime}$, but using the fusion rules of the $\mathrm{SU}(2)$ Lie algebra, not the $\mathrm{SU}(2)_{3} \mathrm{KM}$ algebra. Heuristically, the difference-KM versus Lie-between the algebras governing orbital and spin fusion reflects the fact that the bath and impurity are coupled in the orbital sector, where the bath "absorbs" the impurity orbital isospin, but decoupled in the spin sector, where the impurity spin remains a spectator. 


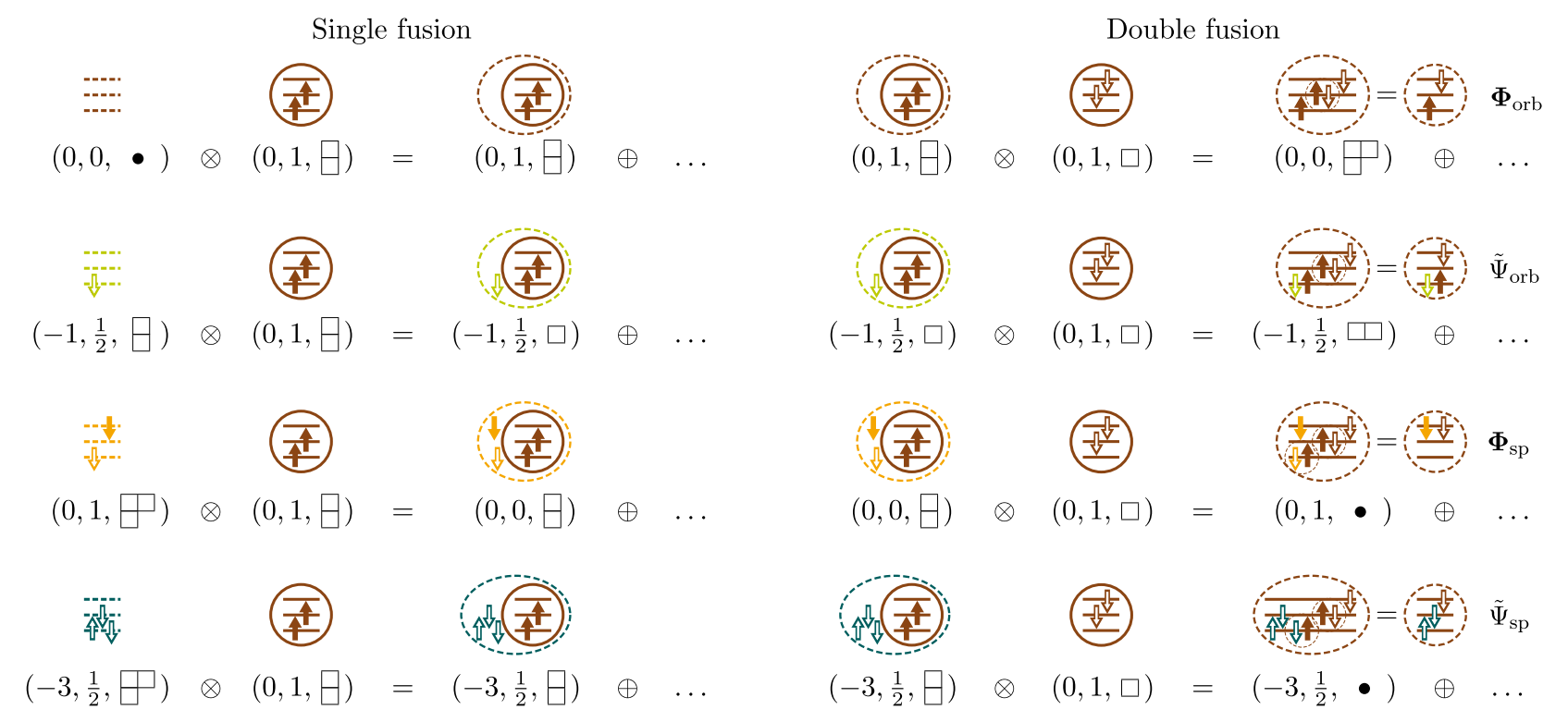

FIG. 3. Schematic depiction of single fusion (left) and double fusion (right), for the four multiplets giving rise to the boundary operators $\boldsymbol{\Phi}_{\text {orb }}, \boldsymbol{\Phi}_{\text {sp }}, \tilde{\Psi}_{\text {orb }}, \tilde{\Psi}_{\text {sp }}$ discussed in Sec. IV (corresponding to rows 1, 3, 4, 5 in Table I). Filled arrows represent electrons, empty arrows represent holes. An electron with spin $\uparrow$ and a hole with spin $\Downarrow$ (missing electron with spin $\uparrow$ ) can be combined to annihilate each other, as indicated by small dashed circles in the last column. Our illustrations depict the impurity using a fermionic representation, as would be appropriate for the 3oAH model, even though the $3 \mathrm{soK}$ impurity has no charge dynamics. In the "single fusion" column, excitations of the free bath are fused with the impurity, $Q_{\mathrm{imp}}=(0,1, \boxminus)$, to obtain the eigenmultiplets of the full system at the NFL fixed point. In the "double fusion" column (right), the single-fusion results are fused with the conjugate impurity representation, $\bar{Q}_{\text {imp }}=(0,1, \square)$. Each of the resulting multiplets is associated with a boundary operator having the same quantum numbers. Colors relate the multiplets obtained after single fusion to the corresponding lines in Fig. 2.

The fusion of bath and impurity degrees of freedom, called single fusion by AL, is illustrated schematically in the left-hand part of Fig. 3 for four selected multiplets. Table II gives a comprehensive list of low-lying multiplets obtained in this manner. On the left it enumerates the 14 lowest-lying multiplets, $(q, S, \lambda)$, of the free bath, with dimensions $d$ and energies $E(q, S, \lambda)$. Fusing these with a $Q_{\text {imp }}=(0,1, \boxminus)$ impurity yields the multiplets $\left(q, S^{\prime}, \lambda^{\prime}\right)$ listed in the center. Their energies are given by $E^{\prime}=E\left(q, S, \lambda^{\prime}\right)$, not $E\left(q, S^{\prime}, \lambda^{\prime}\right)$, since at the NFL fixed point, where $J_{0}=I_{0}=0$, the impurity spin is decoupled from the bath.

The single-fusion excitation energies, $\delta E^{\prime}=E^{\prime}-E_{\min }^{\prime}$, relative to the lowest-lying multiplet $\left(E_{\min }^{\prime}=7 / 30\right)$ are in good agreement (deviations $\lesssim 10 \%$ ) with the values, $E_{\mathrm{NRG}}$, found by NRG (for $K_{0}=0.3, J_{0}=I_{0}=0$ ) for multiplets with corresponding quantum numbers. The agreement improves upon decreasing the NRG discretization parameter $\Lambda$ (here $\Lambda=2.5$ was used). This remarkable agreement between CFT predictions and NRG confirms the applicability of the $\mathrm{SU}(2) \otimes \mathrm{SU}(3)_{2}$ fusion hypothesis proposed above.

(C3) As mentioned in Sec. IV, the fixed point $\mathbf{c}_{\mathrm{NFL}}^{*}$ is characterized by a set of local operators, called boundary operators by AL (since they live at the impurity site, i.e., at the boundary of the two-dimensional space-time on which the CFT is defined). These can be obtained by a second fusion step, called double fusion by AL: the multiplets $\left(q, S^{\prime}, \lambda^{\prime}\right)$ obtained from single fusion are fused with the conjugate impurity representation, $\bar{Q}_{\mathrm{imp}}=(0,1, \square)$, to obtain another set of multiplets, $\left(q, S^{\prime \prime}, \lambda^{\prime \prime}\right)$, listed on the right-hand side of Fig. 3 and Table II. (The conjugate impurity representation has to be used for double fusion to ensure that the set of boundary operators contains the identity operator, $\bar{\lambda} \otimes \lambda=\mathbb{1}$.) Each such multiplet is associated with a boundary operator $\hat{O}$ with the same quantum numbers and scaling dimension, $\Delta=E^{\prime \prime}=E\left(q, S, \lambda^{\prime \prime}\right)$. The operators called $\boldsymbol{\Phi}_{\text {orb }}$ and $\boldsymbol{\Phi}_{\text {sp }}$ are the leading boundary operators (with smallest scaling dimension) in the orbital and spin sectors, respectively. They determine the behavior of the orbital and spin susceptibilities in the NFL regime (see Sec. V C). In the spin-splitting regime, their role is taken by the operators $\tilde{\Psi}_{\text {orb }}$ and $\tilde{\Psi}_{\text {sp }}$, respectively, as discussed in Sec. IV.

\section{Scaling behavior of the susceptibilities}

In this section, we compute the leading frequency dependence of the dynamical spin and orbital susceptibilities. We begin with the NFL regime, where we directly follow the strategy used by AL in Sec. 3.3 of Ref. [44] and show how it reproduces the results presented in Sec. IV. Thereafter we discuss the SS regime, which has no analog in AL's work, using somewhat more heuristic arguments. 


\section{NFL regime}

At the NFL fixed point, the impurity's orbital isospin $\mathbf{T}$ has been fully absorbed into the bath orbital current $\mathcal{J}_{\text {orb }}$ [cf. Eq. (13)]. From this perspective, the impurity orbital susceptibility $\chi_{\text {orb }}^{\text {imp }}$ is governed by the leading local perturbation of the bulk orbital susceptibility, $\chi_{\text {orb }}^{\text {bulk }} \sim$ $\left\langle\mathcal{J}_{\text {orb }}^{\text {bulk }} \| \mathcal{J}_{\text {orb }}^{\text {bulk }}\right\rangle_{\omega}$, where $\quad \mathcal{J}_{\text {orb }}^{\text {bulk }}(t)=\int_{-\infty}^{\infty} \mathrm{d} x \mathcal{J}_{\text {orb }}(t, x) \sim$ $\mathcal{J}_{\text {orb }, n=0}$ is the bulk orbital current. The leading local perturbations are those combinations of boundary operators (found via double fusion; see Table II) having the smallest scaling dimensions and the same symmetry as the bare Hamiltonian [43-45].

In the orbital sector, the leading boundary operator is $\boldsymbol{\Phi}_{\text {orb }}$, with quantum numbers $(0,0, \square)$ and scaling dimension $\Delta_{\text {orb }}=\frac{3}{5}$ (cf. Tables I and II). The orbital current $\mathcal{J}_{\text {orb }}$ has the same quantum numbers. Its first descendant $\mathcal{J}_{\text {orb,-1 }}$ can be combined with $\boldsymbol{\Phi}_{\text {orb }}$ to obtain an orbital SU(3) singlet boundary operator, $H_{\text {orb }}^{\prime}=\mathcal{J}_{\text {orb },-1} \cdot \boldsymbol{\Phi}_{\text {orb }}$, with scaling dimension $1+\Delta_{\text {orb }}=1+\frac{3}{5}$. This is the leading irrelevant (dimension $>1$ ) boundary perturbation to the fixed-point Hamiltonian in the orbital sector. Its contribution to the impurity orbital susceptibility, $\chi_{\text {orb }}^{\text {imp }} \sim \chi_{\text {orb }}^{\text {bulk }}$, evaluated perturbatively to second order, is

$$
\begin{aligned}
\chi_{\text {orb }}^{\text {imp }}(\omega) \sim & \int_{-\infty}^{\infty} d t_{1} \int_{-\infty}^{\infty} d t_{2} \int_{-\infty}^{\infty} d t_{3} e^{i \omega t_{1}} \\
& \times\left\langle\mathcal{J}_{\text {orb }}^{\text {bulk }}\left(t_{1}\right) \cdot \mathcal{J}_{\text {orb }}^{\text {bulk }}(0) H_{\text {orb }}^{\prime}\left(t_{2}\right) H_{\text {orb }}^{\prime}\left(t_{3}\right)\right\rangle \\
\sim & \omega^{2 \Delta_{\text {orb }}-1}=\omega^{1 / 5} .
\end{aligned}
$$

The last line follows by power counting $\left(\mathcal{J}_{\text {orb }}^{\text {bulk has }}\right.$ dimension 0 , each time integral dimension -1 ).

The local bath site coupled to the impurity will show the same behavior, $\chi_{\text {orb }}^{\text {bath }} \sim \omega^{1 / 5}$, since the orbital exchange interaction strongly couples its orbital isospin $\mathbf{J}_{\text {orb }}$ to $\mathbf{T}-$ indeed, $\mathcal{J}_{\text {orb }}$ is constructed from a linear combination of both these operators [cf. Eq. (13)].

The above results can be obtained in a more direct way by positing that at the NFL fixed point, orbital screening causes both $\mathbf{T}$ and $\mathbf{J}_{\text {orb }}$ to be renormalized into the same boundary operator $\boldsymbol{\Phi}_{\text {orb }}$. We then obtain

$$
\chi_{\mathrm{orb}}^{\mathrm{imp}}(\omega) \sim \chi_{\mathrm{orb}}^{\mathrm{bath}}(\omega) \sim\left\langle\boldsymbol{\Phi}_{\mathrm{orb}} \| \boldsymbol{\Phi}_{\text {orb }}\right\rangle_{\omega} \sim \omega^{2 \Delta_{\text {orb }}-1},
$$

reproducing Eq. (14). This is the argument presented in Sec. IV.

We next turn to the spin sector. Exactly at the NFL fixed point, where $J_{0}=I_{0}=0$, the impurity spin $\mathbf{S}$ is decoupled from the bath. At $\mathbf{c}_{\mathrm{NFL}}^{*}$ it hence has no dynamics, scaling dimension 0 , and a trivial spin susceptibility, $\chi_{\mathrm{sp}}^{\mathrm{imp}}(\omega) \sim \delta(\omega)$. By contrast, $\chi_{\mathrm{sp}}^{\text {bath }}$, the susceptibility of $\mathbf{J}_{\mathrm{sp}}$, the local bath spin coupled to the impurity, does show nontrivial dynamics at the fixed point. The reason is that some of the boundary operators induced by orbital screening actually live in the spin sector (a highly nontrivial consequence of non-Abelian bosonization and orbital fusion). The leading boundary operator in the spin sector is $\boldsymbol{\Phi}_{\mathrm{sp}}$, with quantum numbers $(0,1, \bullet)$ and scaling dimension $\Delta_{\mathrm{sp}}=\frac{2}{5}$ (cf. Tables I and II). It can be combined with the first descendant of the (bare, unshifted) spin current to obtain a spin $\mathrm{SU}(2)$ singlet boundary operator, $H_{\mathrm{sp}}^{\prime}=\mathbf{J}_{\mathrm{sp},-1} \cdot \boldsymbol{\Phi}_{\mathrm{sp}}$, with scaling dimension $1+\Delta_{\mathrm{sp}}=$ $1+\frac{2}{5}$. This is the leading irrelevant boundary perturbation to the fixed-point Hamiltonian in the spin sector. Its contribution to the local bath spin susceptibility, $\chi_{\mathrm{sp}}^{\text {bath }} \sim \chi_{\mathrm{sp}}^{\text {bulk }}$, evaluated to second order, is

$$
\begin{aligned}
\chi_{\mathrm{sp}}^{\mathrm{bath}}(\omega) \sim & \int_{-\infty}^{\infty} d t_{1} \int_{-\infty}^{\infty} d t_{2} \int_{-\infty}^{\infty} d t_{3} e^{i \omega t_{1}} \\
& \times\left\langle\mathbf{J}_{\mathrm{sp}}^{\mathrm{bulk}}\left(t_{1}\right) \cdot \mathbf{J}_{\mathrm{sp}}^{\mathrm{bulk}}(0) H_{\mathrm{sp}}^{\prime}\left(t_{2}\right) H_{\mathrm{sp}}^{\prime}\left(t_{3}\right)\right\rangle \\
\sim & \omega^{2 \Delta_{\mathrm{sp}}-1}=\omega^{-1 / 5} .
\end{aligned}
$$

This result, too, can be obtained more directly, by positing that $\mathbf{J}_{\mathrm{sp}}$ is renormalized to $\boldsymbol{\Phi}_{\mathrm{sp}}$, with

$$
\chi_{\mathrm{sp}}^{\mathrm{bath}}(\omega) \sim\left\langle\boldsymbol{\Phi}_{\mathrm{sp}} \| \boldsymbol{\Phi}_{\mathrm{sp}}\right\rangle_{\omega} \sim \omega^{2 \Delta_{\mathrm{sp}}-1},
$$

as argued in Sec. IV.

If the system is tuned very slightly away from the NFL fixed point, $J_{0} \ll 1, I_{0}=0$, the impurity spin does acquire nontrivial dynamics, due to the action of the spin exchange interaction, $J_{0} \mathbf{S} \cdot \mathbf{J}_{\mathrm{sp}}$. According to the above argument, orbital screening renormalizes it to $J_{0} \mathbf{S} \cdot \boldsymbol{\Phi}_{\mathrm{sp}}$. Its secondorder contribution to the impurity spin susceptibility is

$$
\begin{aligned}
\chi_{\mathrm{sp}}^{\mathrm{imp}}(\omega) \sim & \int_{-\infty}^{\infty} d t_{1} \int_{-\infty}^{\infty} d t_{2} \int_{-\infty}^{\infty} d t_{3} e^{i \omega t_{1}} \\
& \times\left\langle\mathbf{S}\left(t_{1}\right) \cdot \mathbf{S}(0)\left(\mathbf{S} \cdot \boldsymbol{\Phi}_{\mathrm{sp}}\right)\left(t_{2}\right)\left(\mathbf{S} \cdot \boldsymbol{\Phi}_{\mathrm{sp}}\right)\left(t_{3}\right)\right\rangle \\
\sim & \omega^{2 \Delta_{\mathrm{sp}}-3}=\omega^{-11 / 5} .
\end{aligned}
$$

The occurrence of such a large, negative exponent for the spin susceptibility is very unusual. It reflects the fact that near (but not at) the NFL fixed point the impurity spin is almost (but not fully) decoupled from the bath, and hence able to "sense" that orbital screening modifies the bath spin current in a nontrivial manner.

\section{Spin-slitting regime}

The renormalized exchange interaction $J_{0} \mathbf{S} \cdot \boldsymbol{\Phi}_{\mathrm{sp}}$ is a relevant perturbation, with scaling dimension $\frac{2}{5}<1$. It grows under the RG flow, eventually driving the system away from the NFL fixed point and into a crossover regime, $T_{\mathrm{sp}}<\omega<T_{\mathrm{ss}}$, called the spin-splitting regime in Sec. III. In the NRG flow diagram of Fig. 2(a), this regime is characterized by level crossings, extending over several 

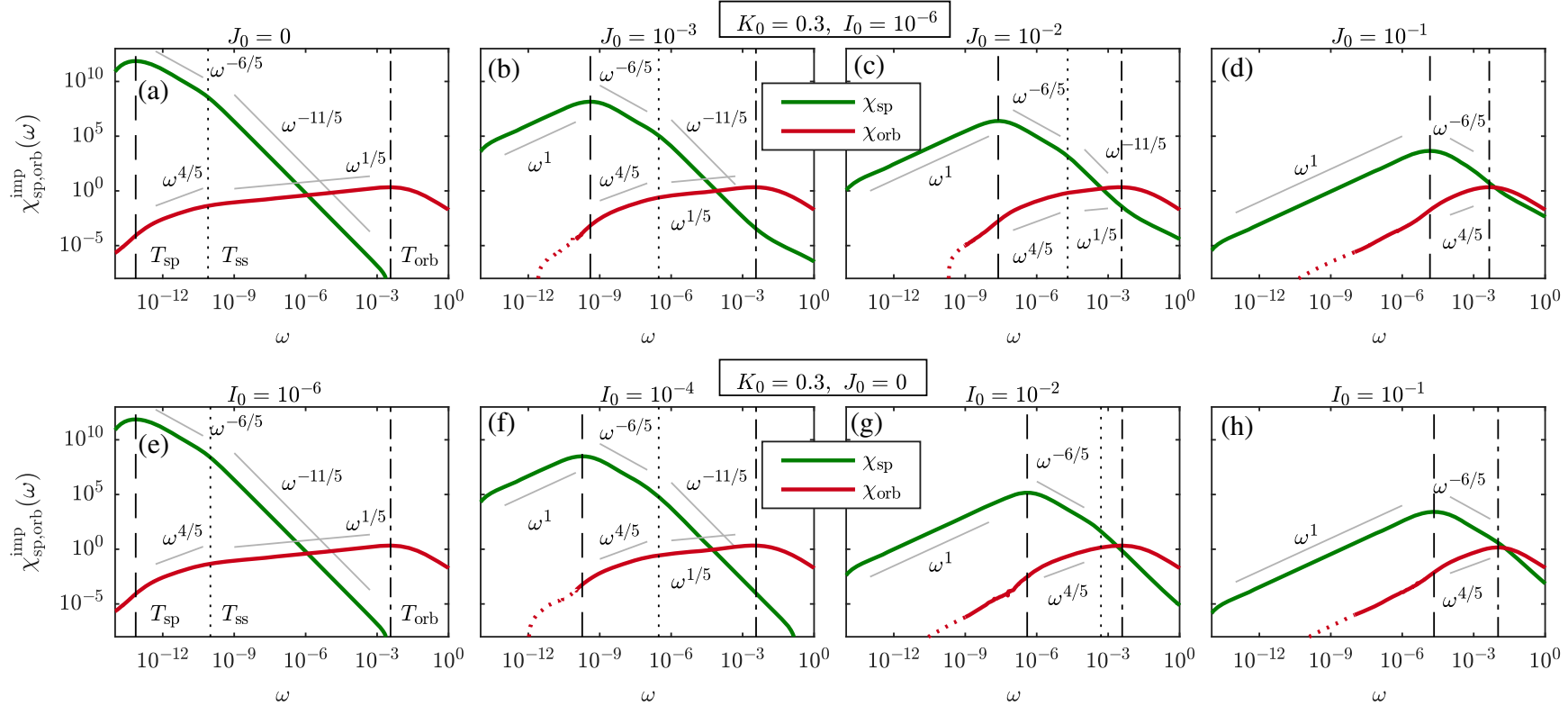

FIG. 4. Imaginary part of the zero-temperature impurity spin and orbital susceptibilities for the 3 soK model. We keep $K_{0}=0.3$ fixed throughout, and (a)-(d) vary $J_{0}$ at fixed $I_{0}=10^{-6}$, or (e)-(h) vary $I_{0}$ at fixed $J_{0}=0$. (a)-(d) As $J_{0}$ is increased from 0 (left) to $10^{-1}$ (right), the width of the NFL regime $\left[T_{\mathrm{ss}}, T_{\mathrm{orb}}\right]$ shrinks, while that of the SS regime $\left[T_{\mathrm{sp}}, T_{\mathrm{ss}}\right]$ remains constant. (e)-(h) We find the same behavior when increasing $I_{0}$ with $K_{0}$ and $J_{0}$ kept fixed.

orders of magnitude in energy, rather than a stationary level structure. Hence the SS regime cannot be characterized by proximity to some well-defined fixed point. (A stationary level structure, characteristic of a FL fixed point, emerges only after another crossover, setting in at the scale $T_{\mathrm{sp}}$.) Nevertheless, Figs. 2(c) and 2(d) show that the local orbital and spin susceptibilities $d o$ exhibit well-defined power-law behavior in the SS regime:

$$
\chi_{\text {orb }}^{\text {imp,bath }}(\omega) \sim \omega^{4 / 5}, \quad \chi_{\mathrm{sp}}^{\text {imp,bath }}(\omega) \sim \omega^{-6 / 5} .
$$

We define the width of the SS regime as the energy range showing this behavior. It extends over about 3 orders of magnitude, independent of $J_{0}$ and $I_{0}$-increasing either of these couplings rigidly shifts the SS regime to larger energies without changing its width (see Fig. 4); i.e., the ratio $T_{\mathrm{sp}} / T_{\mathrm{ss}}$ is independent of these couplings.

The latter fact leads us to conjecture that the NFL fixed point does, after all, govern the SS regime too, though "from afar" rather than from up close. In technical terms, we conjecture that the leading behavior in the SS regime is governed by two different boundary operators, $\tilde{\Psi}_{\text {orb }}$ and $\tilde{\Psi}_{\text {sp }}$, with scaling dimensions $\tilde{\Delta}_{\text {orb }}=\tilde{\Delta}_{\text {sp }}=\frac{9}{10}$ (cf. Tables I and II) instead of the boundary operators $\boldsymbol{\Phi}_{\text {orb }}$ and $\boldsymbol{\Phi}_{\mathrm{sp}}$ governing the NFL regime. This conjecture is encoded in the equation above Eq. (10). It states that $\mathbf{J}_{\text {orb }}$ and $\mathbf{T}$ are both renormalized to $\tilde{\Psi}_{\text {orb }}$, causing $\chi_{\text {orb }}^{\text {bath }}$ and $\chi_{\text {orb }}^{\text {imp }}$ to scale with the same power,

$$
\chi_{\text {orb }}^{\text {bath,imp }} \sim\left\langle\tilde{\Psi}_{\text {orb }} \| \tilde{\Psi}_{\text {orb }}\right\rangle_{\omega} \sim \omega^{2 \tilde{\Delta}_{\text {orb }}-1}=\omega^{4 / 5},
$$

and that $\mathbf{J}_{\mathrm{sp}}$ and $\mathbf{S}$ are both renormalized to $\mathbf{S}+\tilde{\Psi}_{\mathrm{sp}}$, causing $\chi_{\mathrm{sp}}^{\text {bath }}$ and $\chi_{\mathrm{sp}}^{\mathrm{imp}}$ to scale with the same power,

$$
\chi_{\mathrm{sp}}^{\text {bath,imp }} \sim\left\langle\tilde{\Psi}_{\mathrm{sp}} \| \tilde{\Psi}_{\mathrm{sp}}\right\rangle_{\omega} \sim \omega^{2 \tilde{\Delta}_{\mathrm{sp}}-3}=\omega^{-6 / 5} .
$$

The latter result is obtained in a manner analogous to Eq. (18), with $\mathbf{S} \cdot \boldsymbol{\Phi}$ replaced by $\mathbf{S} \tilde{\Psi}_{\text {sp }}[60]$.

\section{Impurity spectral function}

We next consider the leading frequency dependence of the impurity spectral function in the NFL regime. For a Kondo-type impurity, this function is given by $-(1 / \pi) \operatorname{Im} \mathcal{T}(\omega)$, where $\mathcal{T}(\omega)=\left\langle O_{m \sigma} \| O_{m \sigma}^{\dagger}\right\rangle_{\omega}$ is the impurity $\mathcal{T}$ matrix, with $O_{m \sigma}=\left[\psi_{m \sigma}, H_{\text {int }}\right]$ [63].

As discussed in Sec. VC1, the leading irrelevant boundary operators in the NFL regime are $H_{\text {orb }}^{\prime}=\mathcal{J}_{\text {orb,-1 }}$. $\boldsymbol{\Phi}_{\text {orb }}$ and $H_{\mathrm{sp}}^{\prime}=\mathbf{J}_{\mathrm{sp},-1} \cdot \boldsymbol{\Phi}_{\mathrm{sp}}$, with scaling dimensions $1+$ $\Delta_{\text {orb }}=1+\frac{3}{5}$ and $1+\Delta_{\text {sp }}=1+\frac{2}{5}$, respectively. AL have shown that a boundary perturbation of this type, with dimension $1+\Delta$, causes the $\mathcal{T}$ matrix to acquire a leading frequency dependence of $\operatorname{Im} \mathcal{T} \sim|\omega|^{\Delta}$ [45].

For $\omega>0$, our NRG results are consistent with $\operatorname{Im} \mathcal{T} \sim$ $\omega^{3 / 5}$ (cf. Fig. 5). This suggests that the prefactor of $H_{\text {orb }}^{\prime}$ is much larger than that of $H_{\mathrm{sp}}^{\prime}$, presumably because the computation was done for $J_{0}=I_{0}=0$. For $\omega<0$, by contrast, our numerical results do not exhibit clear power-law 


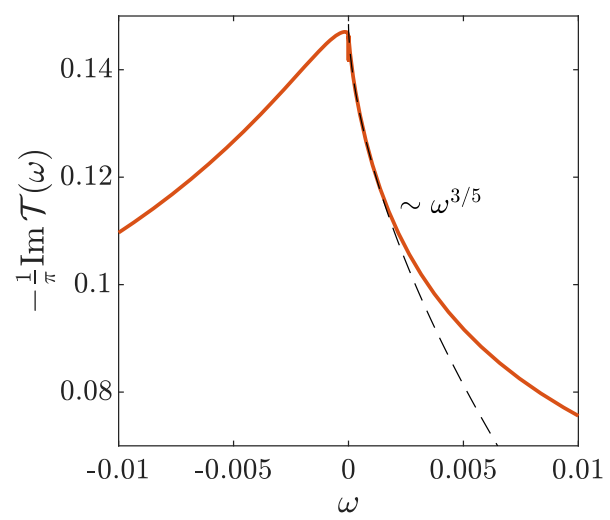

FIG. 5. Impurity spectral function, computed by FDM NRG [52], for $\left(J_{0}, K_{0}, I_{0}\right)=(0,0.3,0)$. For $\omega>0$, the $\omega^{3 / 5}$ behavior is consistent with a boundary perturbation given by $H_{\text {orb }}^{\prime}$. For $\omega<0$, clear power law cannot be determined.

behavior for small $|\omega|$, implying that $\operatorname{Im} \mathcal{T}$ does not have particle-hole symmetry. This is not surprising: the 3 soK model itself breaks particle-hole symmetry, since under a particle-hole transformation, the impurity's orbital multiplet $\exists$ is mapped to $\square$. We suspect that the prefactor of the $|\omega|^{\Delta_{\text {orb }}}$ contribution to $\operatorname{Im} \mathcal{T}$ vanishes for $\omega<0$ for the impurity orbital representation $\boxminus$, such that only subleading boundary operators, with dimensions $\Delta \geq 9 / 10$ (cf. Table II), determine the small- $\omega$ scaling behavior. However, a detailed understanding of this matter is still lacking.

\section{E. Fermi-liquid fixed point}

In this section we show how the FL spectrum at the fixed point $\mathbf{c}_{\mathrm{FL}}^{*}$ can be derived analytically. This can be done in two complementary ways. The first uses $\mathrm{SU}(2)_{3}$ fusion in the spin sector, the second $\mathrm{SU}(6)_{1}$ fusion in the flavor (combined spin + orbital) sector.

\section{Fermi-liquid spectrum via $\mathrm{SU}(2)_{3}$ fusion}

It is natural to ask whether the FL spectrum at $\mathbf{c}_{\mathrm{FL}}^{*}$ can be derived from the NFL spectrum of $\mathbf{c}_{\mathrm{NFL}}^{*}$ via some type of fusion in the spin sector, reflecting spin screening induced by the spin exchange interaction. For example, we have tried the following simple strategy ("naive spin fusion"): when setting up the fusion table (Table II), the bath and impurity spin degrees of freedom are combined, $S \otimes S_{\text {imp }}=\sum_{\oplus} S^{\prime}$, using the fusion rules of the $\mathrm{SU}(2)_{3} \mathrm{KM}$ algebra (Table $\mathrm{S} 3$ in the SM [54]) instead of the SU(2) Lie algebra, and the orbital degrees of freedom, $\lambda \otimes \lambda_{\text {imp }}=\sum_{\oplus} \lambda^{\prime}$, using SU(3) 2 KM fusion rules (as before; see Table S2 in the SM [54]). Moreover, the energies of the resulting multiplets are computed as $E\left(q, S^{\prime}, \lambda^{\prime}\right)$, not $E\left(q, S, \lambda^{\prime}\right)$. However, this naive spin fusion strategy completely fails to reproduce the FL fixed point spectrum obtained by NRG, yielding completely different excitation energies and degeneracies.

We suspect that this failure is due to the fact that the RG flow does not directly pass from the NFL regime into the FL regime, but first traverses the intermediate SS regime.

TABLE III. Fusion table for spin screening at the FL fixed point $\mathbf{c}_{\mathrm{FL}}^{*}$ of the 3 soK model. It has the same structure as Table II, but here single fusion of bath and impurity multiplets in the charge and spin sectors is performed using $\mathrm{U}(1) \times \mathrm{SU}(2)_{3}$ fusion rules (listed in Table S3 of the SM [54]). Moreover, we choose $Q_{\mathrm{imp}}=\left(1, \frac{3}{2}, \bullet\right)$ for the impurity, representing the effective local degree of freedom obtained after the completion of orbital screening. The resulting multiplets $\left(q^{\prime}, S^{\prime}, \lambda\right)$ have eigenenergies $E^{\prime}=E\left(q^{\prime}, S^{\prime}, \lambda\right)$ and excitation energies $\delta E^{\prime}=E^{\prime}-E_{\min }^{\prime}$. The NRG energies, computed for $\left(J_{0}, K_{0}, I_{0}\right)=\left(10^{-4}, 0.3,0\right)$, have been shifted and rescaled such that the lowest energy is zero and the second-lowest values for $E_{\mathrm{NRG}}$ and $\delta E^{\prime}$ match. The single-fusion and NRG spectra agree very well (deviations $\lesssim 2 \%$ ).

\begin{tabular}{|c|c|c|c|c|c|c|c|c|c|c|c|c|c|c|c|}
\hline \multicolumn{5}{|c|}{ Free fermions } & \multicolumn{6}{|c|}{ Single fusion, with $Q_{\text {imp }}=\left(1, \frac{3}{2}, \bullet\right)$} & \multirow{2}{*}{$\begin{array}{l}\mathrm{NRG} \\
E_{\mathrm{NRG}} \\
\end{array}$} & \multicolumn{4}{|c|}{ Double fusion, with $\bar{Q}_{\text {imp }}=\left(-1, \frac{3}{2}, \bullet\right)$} \\
\hline$q$ & $S$ & $\lambda$ & $d$ & $E$ & $q^{\prime}$ & $S^{\prime}$ & $\lambda^{\prime}$ & $d$ & $E^{\prime}$ & $\delta E^{\prime}$ & & $q^{\prime \prime}$ & $S^{\prime \prime}$ & $\lambda^{\prime \prime}$ & $\Delta$ \\
\hline 0 & 0 & $\cdot$ & 1 & 0 & +1 & $\frac{3}{2}$ & • & 4 & $\frac{5}{6}$ & $\frac{1}{2}$ & 0.50 & 0 & 0 & • & 0 \\
\hline+1 & $\frac{1}{2}$ & $\square$ & 6 & $\frac{1}{2}$ & +2 & 1 & $\square$ & 9 & 1 & $\frac{2}{3}$ & 0.67 & +1 & $\frac{1}{2}$ & $\square$ & $\frac{1}{2}$ \\
\hline-1 & $\frac{1}{2}$ & $\boxminus$ & 6 & $\frac{1}{2}$ & 0 & 1 & $\boxminus$ & 9 & $\frac{2}{3}$ & $\frac{1}{3}$ & 0.33 & -1 & $\frac{1}{2}$ & $\boxminus$ & $\frac{1}{2}$ \\
\hline 0 & 1 & $\exists$ & 24 & 1 & +1 & $\frac{1}{2}$ & $\square$ & 16 & $\frac{5}{6}$ & $\frac{1}{2}$ & 0.50 & 0 & 1 & $\square$ & 1 \\
\hline+2 & 0 & $\square$ & 6 & 1 & +3 & $\frac{3}{2}$ & $\square$ & 24 & $\frac{13}{6}$ & $\frac{11}{6}$ & 1.87 & +2 & 0 & $\square$ & 1 \\
\hline-2 & 0 & $\boxplus$ & 6 & 1 & -1 & $\frac{3}{2}$ & $\bigoplus$ & 24 & $\frac{3}{2}$ & $\frac{7}{6}$ & 1.16 & -2 & 0 & $\bigoplus$ & 1 \\
\hline+2 & 1 & $\square$ & 9 & 1 & +3 & $\frac{1}{2}$ & $\boxminus$ & 6 & $\frac{7}{6}$ & $\frac{5}{6}$ & 0.84 & +2 & 1 & $\boxminus$ & 1 \\
\hline-2 & 1 & $\square$ & 9 & 1 & -1 & $\frac{1}{2}$ & $\square$ & 6 & $\frac{1}{2}$ & $\frac{1}{6}$ & 0.17 & -2 & 1 & $\square$ & 1 \\
\hline+1 & $\frac{3}{2}$ & $\boxminus$ & 24 & $\frac{3}{2}$ & +2 & 0 & $\boxminus$ & 6 & 1 & $\frac{2}{3}$ & 0.68 & +1 & $\frac{3}{2}$ & $\boxminus$ & $\frac{3}{2}$ \\
\hline-1 & $\frac{3}{2}$ & $\square$ & 24 & $\frac{3}{2}$ & 0 & 0 & $\square$ & 6 & $\frac{2}{3}$ & $\frac{1}{3}$ & 0.34 & -1 & $\frac{3}{2}$ & $\square$ & $\frac{3}{2}$ \\
\hline \pm 3 & $\frac{1}{2}$ & 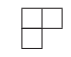 & 16 & $\frac{3}{2}$ & -2 & 1 & $\boxminus$ & 24 & $\frac{4}{3}$ & 1 & 0.99 & -3 & $\frac{1}{2}$ & $\square$ & $\frac{3}{2}$ \\
\hline \pm 3 & $\frac{3}{2}$ & • & 4 & $\frac{3}{2}$ & -2 & 0 & • & 1 & $\frac{1}{3}$ & 0 & 0.00 & -3 & $\frac{3}{2}$ & • & $\frac{3}{2}$ \\
\hline
\end{tabular}


In the latter, the degeneracy between the two degenerate ground state multiplets of the NFL regime, $\left(1, \frac{1}{2}, \bullet\right)$ and $\left(1, \frac{3}{2}, \bullet\right)$, is lifted, in a manner that seems to elude a simple description via a modified spin fusion rule.

Instead, the FL spectrum can be obtained via the following arguments. The ground state multiplet of the SS regime, $\left(1, \frac{3}{2}, \bullet\right)$, describes an effective local degree of freedom coupled to a bath in such a manner that one bath electron fully screens the impurity orbital isospin, while their spins add to a total spin of $\frac{1}{2}+1=\frac{3}{2}$ [see Fig. 2(b)]. Let us view this as an effective impurity with $Q_{\text {imp }}=$ $\left(1, \frac{3}{2}, \bullet\right)$. If we combine its charge and spin degrees of freedom with those of a free bath, using $q+q_{\text {imp }}=q^{\prime}$ and $S \oplus S_{\text {imp }}=\sum_{\oplus} S^{\prime}$, fused according to the $\mathrm{SU}(2)_{3} \mathrm{KM}$ algebra, the resulting single-fusion spectrum fully reproduces the FL spectrum found by NRG, as shown in Table III.

\section{Fermi-liquid spectrum via $\mathrm{SU}(6)_{1}$ fusion}

The FL ground state of the 3 soK model is a fully screened spin and orbital singlet. It is therefore natural to expect that the FL spectrum has a higher symmetry, namely that of the group $\mathrm{U}(1) \times \mathrm{SU}(6)$, which treats spin and orbital excitations on an equal footing.

This is indeed the case: we now show that the FL spectrum of the 3 soK model discussed above matches that of an SU(6) Kondo model which does not distinguish between spin and orbital degrees of freedom. We consider a bath with six flavors of electrons, $H_{\text {bath }}=$ $\sum_{p} \sum_{\nu=1}^{6} \varepsilon_{p} \psi_{p \nu}^{\dagger} \psi_{p \nu}$ and an impurity-bath coupling of the form $J_{U} \mathbf{U} \cdot \mathbf{J}_{\mathrm{fl}}$. Here $\mathbf{J}_{\mathrm{fl}}$ is the flavor density at the impurity site, with $J_{\mathrm{fl}}^{a}=\psi_{\nu}^{\dagger} \frac{1}{2} \lambda_{\nu \nu^{\prime}}^{a} \psi_{\nu^{\prime}}$, where $\lambda^{a}$ are SU(6) matrices in the fundamental representation, and $\mathbf{U}$ describes the impurity's SU(6) flavor isospin, chosen in the fully antisymmetric representation $\boxminus$. The latter has dimension 15 , representing the $\left(\begin{array}{l}6 \\ 2\end{array}\right)$ ways of placing two identical particles into six available states.

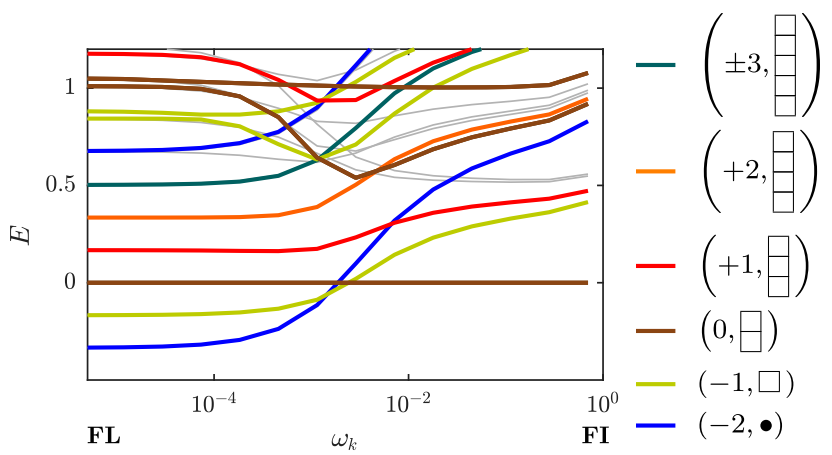

FIG. 6. NRG results for the $\mathrm{SU}(6)$ Kondo model with $J_{U}=0.1$, shown using $\mathcal{E}_{\text {ref }}=\mathcal{E}(0, \boxminus)$ as reference energy. The computation was performed using QSpace [50] to exploit the model's full $\mathrm{U}(1) \times \mathrm{SU}(6) \quad$ symmetry. $\quad(\mathrm{NRG}$ parameters: $\Lambda=2.5$, $N_{\text {keep }}=2000, D=1$.)
Figure 6 shows the NRG finite-size eigenlevel flow for this model. It exhibits a single crossover from a free-impurity fixed point, with ground state multiplet $(q, \lambda)=(0, \boxminus)$, to a FL fixed point, whose ground state multiplet $(-2, \bullet)$ involves complete screening of the impurity's flavor isospin degree of freedom.

This crossover can be described analytically by using non-Abelian bosonization followed by flavor fusion. We begin by using non-Abelian bosonization with the $\mathrm{U}(1) \times$ $\mathrm{SU}(6)_{1} \mathrm{KM}$ current algebra to express the bath excitation spectrum in the form

$$
\begin{aligned}
E(q, \lambda)= & \frac{1}{12} q^{2}+\frac{1}{7} \kappa_{6}(\lambda)+\ell, \\
\kappa_{6}(\lambda)= & \frac{1}{12}\left(5 \lambda_{1}^{2}+8 \lambda_{2}^{2}+9 \lambda_{3}^{2}+8 \lambda_{4}^{2}+5 \lambda_{5}^{2}\right) \\
& +\frac{1}{2}\left(5 \lambda_{1}+8 \lambda_{2}+9 \lambda_{3}+8 \lambda_{4}+5 \lambda_{5}\right) \\
& +\frac{1}{6}\left(6 \lambda_{2} \lambda_{3}+6 \lambda_{3} \lambda_{4}+4 \lambda_{1} \lambda_{2}+4 \lambda_{2} \lambda_{4}+4 \lambda_{4} \lambda_{5}\right. \\
& \left.+3 \lambda_{1} \lambda_{3}+3 \lambda_{3} \lambda_{5}+2 \lambda_{1} \lambda_{4}+2 \lambda_{2} \lambda_{5}+\lambda_{1} \lambda_{5}\right),
\end{aligned}
$$

with $\ell \in \mathbb{Z}$, where $\kappa_{6}(\lambda)$ is the quadratic Casimir for the representation $\lambda=\left(\lambda_{1}, \lambda_{2}, \lambda_{3}, \lambda_{4}, \lambda_{5}\right)$ of the SU(6) Lie algebra [62]. [The contributions from the two terms of Eq. (22a) are listed in Table S4 in the SM [54] for all $q$ and $\lambda$ values needed in Table IV.] The few lowest-lying $(q, \lambda)$

TABLE IV. Fusion table for flavor screening at the FL fixed point of the SU(6) Kondo model. The table has the same structure as the left and center parts of Table II, but here the free bath excitations are labeled $(q, \lambda)$, their energies are computed using Eqs. (22) and Table S4 of the SM [54], and flavor fusion with $Q_{\mathrm{imp}}=(0, \boxminus)$ is performed using $\mathrm{SU}(6)_{1}$ fusion rules (listed in Table S5 of the SM [54]). The resulting multiplets $\left(q, \lambda^{\prime}\right)$ have eigenenergies $E^{\prime}=E\left(q, \lambda^{\prime}\right)$, degeneracies $d^{\prime}$, and excitation energies $\delta E^{\prime}=E^{\prime}-E_{\min }^{\prime}$. The FL spectrum, obtained by $\mathrm{U}(1) \times$

\begin{tabular}{|c|c|c|c|c|c|c|c|c|c|}
\hline \multicolumn{4}{|c|}{ Free fermions } & \multicolumn{5}{|c|}{ Single fusion, $Q_{\mathrm{imp}}=(0, \boxminus)$} & \multirow{2}{*}{$\frac{\mathrm{NRG}}{E_{\mathrm{NRG}}}$} \\
\hline$q$ & $\lambda$ & $d$ & $E$ & $q$ & $\lambda^{\prime}$ & $d^{\prime}$ & $E^{\prime}$ & $\delta E^{\prime}$ & \\
\hline 0 & • & 1 & 0 & 0 & $\theta$ & 15 & $\frac{2}{3}$ & $\frac{1}{3}$ & 0.33 \\
\hline+1 & $\square$ & 6 & $\frac{1}{2}$ & +1 & 日 & 20 & $\frac{5}{6}$ & $\frac{1}{2}$ & 0.50 \\
\hline-1 & $\exists$ & 6 & $\frac{1}{2}$ & -1 & $\square$ & 6 & $\frac{1}{2}$ & $\frac{1}{6}$ & 0.17 \\
\hline+2 & $\square$ & 15 & 1 & +2 & $\theta$ & 15 & 1 & $\frac{2}{3}$ & 0.67 \\
\hline-2 & $\forall$ & 15 & 1 & -2 & $\bullet$ & 1 & $\frac{1}{3}$ & 0 & 0 \\
\hline \pm 3 & $\theta$ & 20 & $\frac{3}{2}$ & \pm 3 & B & 6 & $\frac{7}{6}$ & $\frac{5}{6}$ & 0.84 \\
\hline
\end{tabular}
$\mathrm{SU}(6) \mathrm{NRG}$ calculations (Fig. 6) for $J_{U}=0.1$, is shown on the right. It has been shifted and rescaled such that the lowest energy is zero and the second-lowest values for $E_{\mathrm{NRG}}$ and $\delta E^{\prime}$ match. The single-fusion and NRG spectra agree very well (deviations $\lesssim 1 \%$ ). 

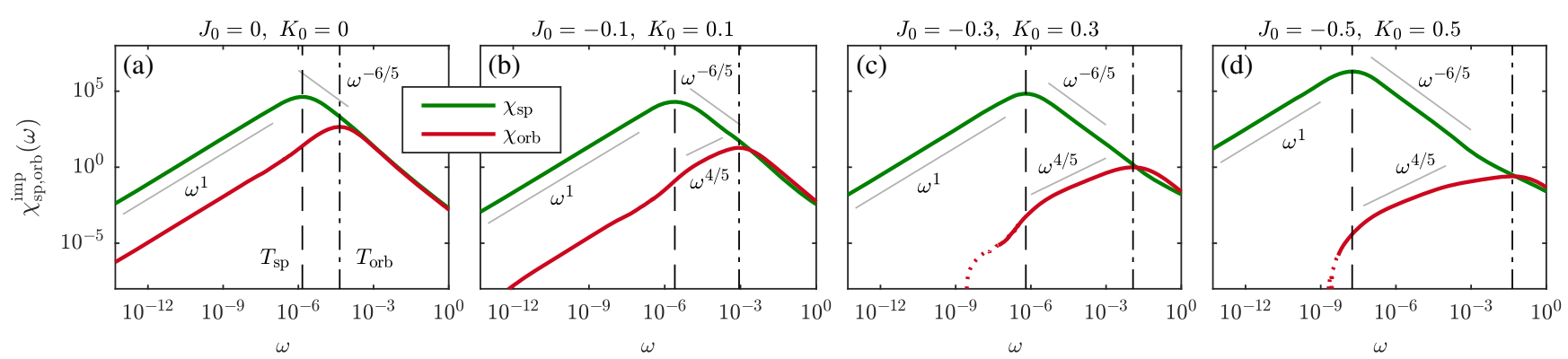

FIG. 7. Imaginary part of the impurity spin and orbital susceptibilities for the Anderson-Kondo model, with $U=5, J_{H}=1, \Gamma=0.1$, $I_{0}=0$, and $J_{0}, K_{0}$ varying from (a) $J_{0}=K_{0}=0$ (pure Anderson-Hund model) to (d) $-J_{0}=K_{0}=0.5$. The energy scales for spin and orbital screening, $T_{\mathrm{sp}}$ and $T_{\text {orb }}$, are pushed apart with increasing $-J_{0}=K_{0}$.

multiplets of the free bath, having $E(q, \lambda) \in \frac{1}{2} \mathbb{Z}$, are listed on the left-hand side of Table IV. The strong-coupling FL spectrum can be obtained by combining the bath and impurity flavor degrees of freedom, $\lambda \otimes \lambda_{\text {imp }}=\sum_{\oplus} \lambda^{\prime}$, using the fusion rules of the $\mathrm{SU}(6)_{1} \mathrm{KM}$ algebra (see Table S5 in the SM [54]). The resulting multiplets $\left(q, \lambda^{\prime}\right)$ are listed in the center of Table IV. Their eigenenergies fully match those from NRG.

\section{THREE-ORBITAL ANDERSON-KONDO MODEL}

The 3 soK model, which we study in detail above, is derived from the more realistic $3 \mathrm{oAH}$ model by a Schrieffer-Wolff transformation. In the following, we explore another route for smoothly connecting the physics of the two models, namely starting from the $3 \mathrm{oAH}$ model and then additionally turning on the spin and orbital exchange couplings of the 3 soK model.

The Hamiltonian of the $3 \mathrm{oAH}$ model [22] has the following form: $H_{\text {bath }}+H_{\text {hyb }}+H_{3 \mathrm{oAH}}$,

$$
\begin{aligned}
H_{3 \mathrm{oAH}} & =\frac{3}{4} J_{H} N_{\mathrm{imp}}+\frac{1}{2}\left(U-\frac{1}{2} J_{H}\right) N_{\mathrm{imp}}\left(N_{\mathrm{imp}}-1\right)-J_{H} \mathbf{S}^{2}, \\
H_{\mathrm{hyb}} & =\sum_{p m \sigma} V_{p}\left(f_{m \sigma}^{\dagger} \psi_{p m \sigma}+\text { H.c. }\right),
\end{aligned}
$$

with the impurity occupation $N_{\text {imp }}=\sum_{m \sigma} f_{m \sigma}^{\dagger} f_{m \sigma}$, where $f_{m \sigma}^{\dagger}$ creates an impurity electron with spin $\sigma$ in orbital $m$. A hybridization function, $\Gamma(\varepsilon)=\pi \sum_{p}\left|V_{p}\right|^{2} \delta\left(\varepsilon-\varepsilon_{p}\right) \equiv$ $\Gamma \Theta(D-|\varepsilon|)$, controls the hopping between the impurity and the bath. $U$ is the local Coulomb interaction and $J_{H}$ the Hund's coupling, favoring a large spin. To this Hamiltonian we add $J_{0} \mathbf{S} \cdot \mathbf{J}_{\mathrm{sp}}+K_{0} \mathbf{T} \cdot \mathbf{J}_{\text {orb }}$, the Kondo-type spin and orbital exchange couplings between impurity and bath from Eq. (1), with $S^{\alpha}=f_{m \sigma}^{\dagger} \frac{1}{2} \sigma_{\sigma \sigma^{\prime}}^{\alpha} f_{m \sigma^{\prime}}, T^{a}=f_{m \sigma}^{\dagger} \frac{1}{2} \tau_{m m^{\prime}}^{a} f_{m^{\prime} \sigma}$. We treat $J_{0}$ and $K_{0}$ as free parameters and use them to "deform" the $3 \mathrm{oAH}$ model in a way that widens the SOS regime between $T_{\text {sp }}$ and $T_{\text {orb }}$.

Figures 7(a)-7(d) show how the spin and orbital susceptibilities change upon increasing $\left|J_{0}\right|$ and $\left|K_{0}\right|$, with
$J_{0}<0$ and $K_{0}>0$. A pure 3oAH model, with $\left(J_{0}, K_{0}\right)=$ $(0,0)$, clearly shows spin-orbital separation, but $T_{\mathrm{sp}}$ and $T_{\text {orb }}$ differ by less than two decades [Fig. 7(a); see also Ref. [22] ]. Though the SOS window is too small to reveal a true power law for $\chi_{\mathrm{sp}}^{\mathrm{imp}}$, the hints of $\omega^{-6 / 5}$ behavior are already discernable. Turning on the additional exchange coupling terms, with $J_{0}<0$ and $K_{0}>0$, causes $T_{\mathrm{sp}}$ to decrease and $T_{\text {orb }}$ to increase, respectively, widening the SOS regime [Figs. 7 (b)-7(d)]. For $\left(J_{0}, K_{0}\right)=(-0.5,0.5)$ it spans more than 6 orders of magnitude, so that clear power laws, $\chi_{\mathrm{sp}}^{\mathrm{imp}} \sim \omega^{-6 / 5}$ and $\chi_{\mathrm{orb}}^{\mathrm{imp}} \sim \omega^{4 / 5}$, become accessible [Fig. 7(d)]. These power laws are consistent with our findings for the spin-splitting regime in Secs. III and V. This scenario is evidently smoothly connected to that of the pure 3 soK model [Fig. 2(c)]. There the absence of charge fluctuations makes it possible to fully turn off the $I_{0}$ contribution implicitly present in the 3oAH model, thereby widening the SOS regime even further and allowing the true NFL regime to be analyzed in detail.

\section{CONCLUSION}

While the main aim of this work was to understand NFL behavior in Hund metals, it has much wider implications, as already indicated in Sec. I. Let us assess these from several perspectives of increasing generality.

(i) We have used NRG and CFT to elucidate the NFL regime of a 3 soK model, fine-tuned such that spin screening sets in at very much lower energies than orbital screening. We were able to analytically compute the scaling behavior of dynamical spin and orbital susceptibilities, finding $\chi_{\text {orb }}^{\text {imp }} \sim \omega^{1 / 5}$, $\chi_{\mathrm{sp}}^{\mathrm{imp}} \sim \omega^{-11 / 5}$ in the NFL regime and $\chi_{\mathrm{sp}}^{\text {imp }} \sim \omega^{-6 / 5}$ in the spin-splitting regime. The main significance of these findings lies in the qualitative physical behavior which they imply. An orbital susceptibility decreasing with an exponent $<1$, rather than the Fermi-liquid exponent 1, indicates that the orbital degrees of freedom, though screened, are still affected by the unscreened spin degrees of freedom. A spin susceptibility diverging as $\omega^{-\gamma}$, with $\gamma>1$, 
indicates anomalously strong spin fluctuations. This seems to be a characteristic property of the incoherent regime of Hund metals. As pointed out in Sec. I, anomalously strong spin fluctuations have direct consequences for theories of the superconducting state of the iron pnictides [14].

(ii) We have uncovered the origin of hints of NFL behavior found previously for a $3 \mathrm{oAH}$ model and related models [1,3-5,8,22-24]. There the spinorbital coupling $I_{0}$ is always nonzero, preventing RG trajectories from closely approaching the NFL fixed point. Nevertheless, even if they pass this fixed point "at a distance," it still leaves traces of NFL behavior for various observables, such as $\chi_{\mathrm{sp}}^{\mathrm{imp}} \sim$ $\omega^{-6 / 5}$ behavior for the imaginary part of the impurity's dynamical spin susceptibility. We showed in Sec. VI how NFL behavior emerges if the $3 \mathrm{oAH}$ model is "deformed" by additionally turning on the spin and orbital exchange couplings of the 3 soK model, thereby adiabatically connecting the SS regime of the 3 soK model to the incoherent regime of the 3oAH model. Furthermore, it has been shown in Ref. [22] that DMFT self-consistency does not significantly influence the behavior of the susceptibilities in the 3oAH model. Thus our conclusions, in particular regarding the prevalence of strong spin fluctuations in the SOS regime, should also apply to DMFT calculations. Indeed, DMFT studies [22,47] of a selfconsistent $3 \mathrm{oAH}$ model contain results for $\chi_{\mathrm{sp}}^{\mathrm{imp}}$ which, in the SOS window, are consistent with the $\omega^{-6 / 5}$ scaling found and explained here for the SS regime.

(iii) Taking a broader perspective, we have provided an analytic solution of a paradigmatic example of a "Hund impurity problem." We were able to address this fundamental type of problem by combining state-of-the-art multiorbital NRG with a suitable generalization of Affleck and Ludwig's CFT approach [42-46].

(iv) Regarding experimental relevance, Hund impurities are of central importance for understanding Hund metals, including almost all $4 d$ and $5 d$ materials, and even in the $5 f$ actinides Hund's coupling is the main cause for electronic correlations. Our work illustrates paradigmatically why hints of NFL physics can generically be expected to arise in such systems. Moreover, tunable Hund impurities can be realized using magnetic molecules on substrates [64] or multilevel quantum dots, raising hopes of tuning Hund impurities in such a way that truly well-developed NFL behavior can be observed experimentally.

\section{ACKNOWLEDGMENTS}

We thank I. Affleck, A. Georges, M. Goldstein, O. Parcollet, E. Sela, and A. Tsvelik for helpful advice and, in particular, I. Brunner for technical advice regarding $\mathrm{SU}(3)_{2}$ fusion rules. E. W., K. M. S., and J. v. D. are supported by the Deutsche Forschungsgemeinschaft under Germany's Excellence Strategy-EXC-2111-390814868, and S.-S. B. L. by Grant No. LE3883/2-1. A. W. was supported by the U.S. Department of Energy, Office of Basic Energy Sciences, under Contract No. DE-SC0012704. G. K. was supported by the National Science Foundation Grant No. DMR-1733071. Y.W. was supported by the U.S. Department of Energy, Office of Science, Basic Energy Sciences as a part of the Computational Materials Science Program through the Center for Computational Design of Functional Strongly Correlated Materials and Theoretical Spectroscopy.

Note added.-Recently, a paper closely related to ours appeared [65], with similar goals, a complementary analysis (using NRG but not CFT), and conclusions consistent with ours.

\section{APPENDIX: YE'S SU(2) $\times$ SU(2) SPIN-ORBITAL KONDO MODEL}

In this appendix, we revisit an $\mathrm{SU}(2) \times \mathrm{SU}(2)$ spinorbital Kondo (2soK) model studied in a pioneering paper by Ye in 1997 [48]. It is a simpler cousin of our 3 soK model, having a Hamiltonian of precisely the same form, with the following differences: the orbital channel index takes only two values, $m=1,2$; the local orbital current $\mathbf{J}_{\text {orb }}$ is defined using Pauli (not Gell-Mann) matrices; and the impurity spin and orbital isospin operators, $\mathbf{S}$ and $\mathbf{T}$, are both $\mathrm{SU}(2)$ generators, in the representation $S=\lambda=\frac{1}{2}$.

In the context of the present study, Ye's paper is of interest because his Kondo impurity likewise features both spin and orbital degrees of freedom. From a conceptual perspective, his and our models differ only in the symmetry group, $\mathrm{SU}(2)$ versus $\mathrm{SU}(3)$ in the orbital sector, and the choice of impurity multiplet, $Q_{\mathrm{imp}}=\left(\frac{1}{2}, \frac{1}{2}\right)$ versus $(1, \exists)$. Moreover, he was able to obtain exact results for his model using Abelian bosonization. Below, we verify that when the NRG and CFT methodology used in the main text is applied to Ye's 2soK model, the results are consistent with his conclusions.

Before proceeding, however, let us also briefly address some differences between Ye's work and ours. Since he uses Abelian bosonization, his approach does not readily generalize to the $\mathrm{U}(1) \times \mathrm{SU}(2) \times \mathrm{SU}(3)$ impurity model considered here. Ye does mention very briefly that some of his results can also be obtained using non-Abelian bosonization, employing simultaneous fusion in the spin and orbital sectors. However, we show below that that fusion scheme is applicable only when particle-hole symmetry is present. This is the case for Ye's application, but not for our 3 soK model. When particle-hole symmetry is absent, the fusion schemes needed for the spin and orbital are subtly different-indeed, clarifying these differences was the conceptually most challenging aspect of our work. Note that the particle-hole asymmetry of our 3 soK model is not a 

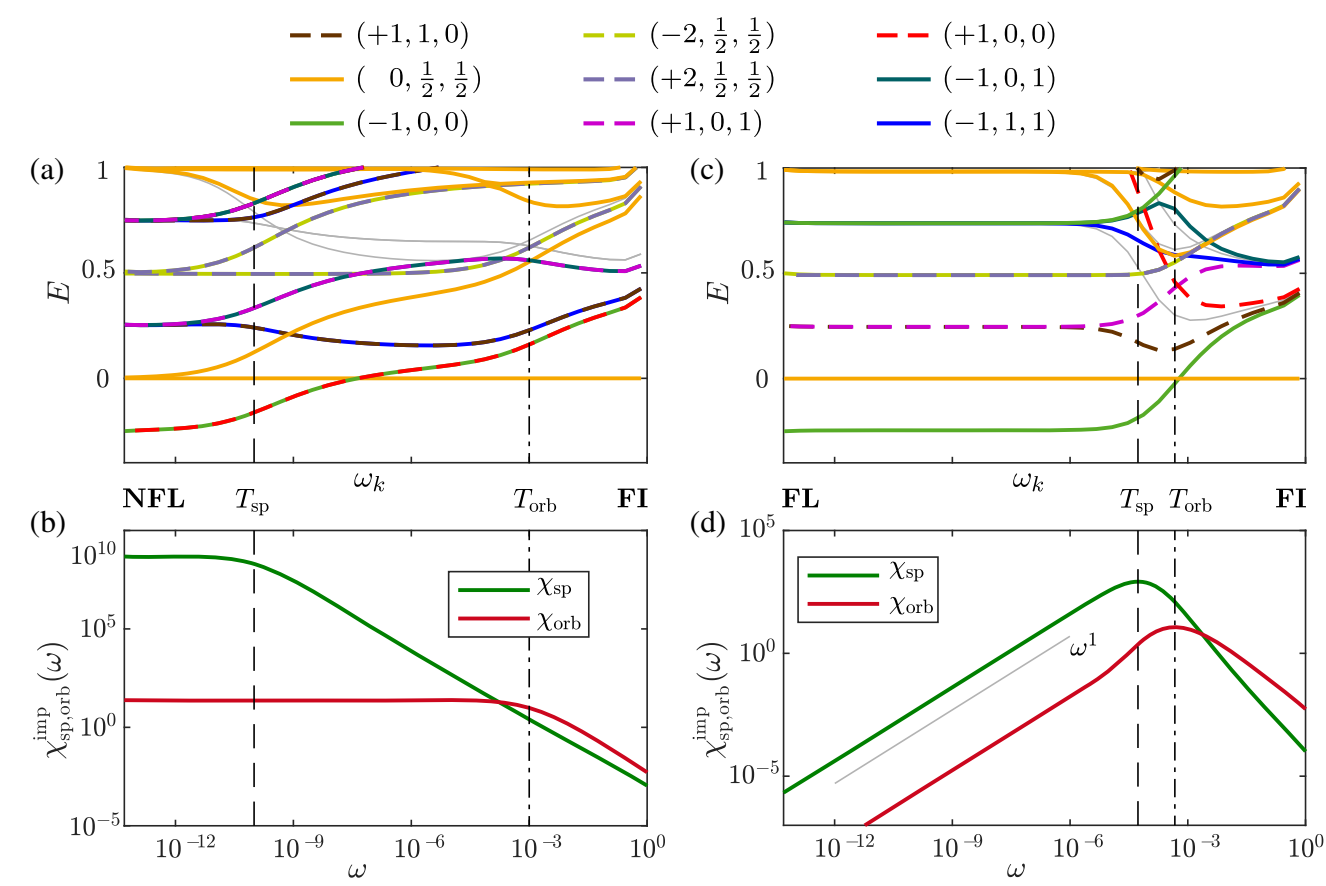

FIG. 8. NRG results for Ye's 2soK model, computed (a),(b) for $\left(J_{0}, K_{0}, I_{0}\right)=(0.1,0.3,0)$, respecting particle-hole symmetry, and (c), (d) for $(0,0.3,0.05)$, breaking particle-hole symmetry. (a),(c) Eigenlevel flow diagrams, with quantum numbers $(q, S, \lambda)$ shown at the top. The low-energy fixed points in (a) and (c) exhibit a NFL or FL spectrum, respectively, reproduced analytically in Table V or VI, respectively. (b),(d) Imaginary part of the impurity's spin and orbital susceptibilities. Vertical lines indicate the crossover scales for orbital screening $T_{\text {orb }}$ (dash-dotted) and spin screening $T_{\text {sp }}$ (dashed), marking when $\chi_{\text {orb }}^{\text {imp }}$ and $\chi_{\mathrm{sp}}^{\text {imp }}$ (b) bend over to constant behavior or (d) reach their maxima, respectively. (NRG parameters: $\Lambda=2.5, N_{\text {keep }}=2000, D=1$.)

mere technical complication, but an essential ingredient of the physics of Hund metals, which typically feature fillings one particle away from half filling. Finally, note that Ye's model, involving an impurity with spin $1 / 2$, is not relevant for Hund metals, where Hund's coupling favors local spins larger than $1 / 2$.

\section{1. $I_{0}=0$ : NFL fixed point}

For $I_{0}=0$, the 2soK model obeys particle-hole symmetry. Figure 8(a) shows the finite-size eigenlevel flow computed by $\mathrm{NRG}$ for $\mathbf{c}_{0}=\left(J_{0}, K_{0}, I_{0}\right)=(0.1,0.3,0)$. The low-energy fixed-point spectrum features equidistant levels, but nevertheless has NFL properties, as predicted by $\mathrm{Ye}$, in that it cannot be understood in terms of combinations of single-particle excitations. Remarkably, this fixed-point spectrum can be reproduced by CFT arguments. Using nonAbelian bosonization according to the $\mathrm{U}(1) \times \mathrm{SU}(2)_{2} \times$ $\mathrm{SU}(2)_{2} \mathrm{KM}$ algebra, the spectrum of free bath excitations can be expressed as

$$
\begin{aligned}
E(q, S, \lambda) & =\frac{1}{8} q^{2}+\frac{1}{4} \kappa_{2}(S)+\frac{1}{4} \kappa_{2}(\lambda)+\ell, \\
\kappa_{2}(S) & =S(S+1), \quad \kappa_{2}(\lambda)=\lambda(\lambda+1),
\end{aligned}
$$

with $\ell \in \mathbb{Z}$, while $\kappa_{2}(S), \kappa_{2}(\lambda)$ are the quadratic $\mathrm{SU}(2)$ Casimirs in the spin and orbital sectors, respectively. We now combine bath and impurity degrees of freedom using simultaneous fusion in the spin and orbital sectors, $S \otimes$ $S_{\text {imp }}=\sum_{\oplus} S^{\prime}$ and $\lambda \otimes \lambda_{\text {imp }}=\sum_{\oplus} \lambda^{\prime}$, employing the fusion rules of the $\mathrm{SU}(2)_{2} \times \mathrm{SU}(2)_{2} \mathrm{KM}$ algebra (Table $\mathrm{S} 7$ in the SM [54]). This reproduces the NFL fixed-point spectrum found by NRG, as shown in Table V.

By contrast, we recall that for the $3 \mathrm{soK}$ model our attempts to use simultaneous spin and orbital fusion to obtain the FL ground state for $0 \neq J_{0} \ll K_{0}, I_{0}=0$ were unsuccessful (cf. Sec. VE 1). Thus the 2 soK and 3 soK models provide an example and a counterexample for the success of simultaneous spin and orbital fusion, succeeding or failing for a NFL or FL fixed point spectrum, respectively.

We have also computed the imaginary parts of spin and orbital susceptibilities $\chi_{\mathrm{sp}, \text { orb }}^{\text {imp }}(\omega)$. Figure $8(\mathrm{~b})$ shows the results. Both functions approach constants in the zerofrequency limit, i.e., scale as $\omega^{0}$. This can be understood in terms of the scaling dimensions of the leading boundary operators in the spin and orbital sectors, $\boldsymbol{\Phi}_{\mathrm{sp}}$ and $\boldsymbol{\Phi}_{\mathrm{orb}}$, which have dimensions $\Delta_{\mathrm{sp}}=\Delta_{\text {orb }}=\frac{1}{2}($ Table V). By the arguments of Sec. V C, we thus obtain

$$
\chi_{\mathrm{sp}, \text { orb }}^{\mathrm{imp}} \sim \omega^{2 \Delta_{\mathrm{sp}, \text { orb }}-1} \sim \omega^{0},
$$

as predicted by Ye. This resembles the behavior observed for the celebrated two-channel Kondo model, featuring a 
TABLE V. Fusion table for the NFL fixed point of Ye's 2soK model. It is structured just as Table II for the 3soK model, but here the free bath excitations are computed using Eqs. (A2) and Table S6 of the SM [54], and single fusion of bath and impurity degrees of freedom is performed simultaneously in the spin and orbital sectors, using $\mathrm{SU}(2)_{2} \times \mathrm{SU}(2)_{2}$ fusion rules (listed in Table $\mathrm{S} 7$ of the SM [54]). The resulting multiplets $\left(q, S^{\prime}, \lambda^{\prime}\right)$ have energies $E^{\prime}=E\left(q, S^{\prime}, \lambda^{\prime}\right)$ and excitation energies $\delta E^{\prime}=E^{\prime}-E_{\text {min }}^{\prime}$. The NRG energies, computed for $\left(J_{0}, K_{0}, I_{0}\right)=(0.1,0.3,0)$ [Fig. 8(a)], have been shifted and rescaled such that the lowest energy is zero and the second-lowest values for $E_{\mathrm{NRG}}$ and $\delta E^{\prime}$ match. The single fusion and NRG spectra agree very well (deviations $\left.\lesssim 1 \%\right)$.

\begin{tabular}{|c|c|c|c|c|c|c|c|c|c|c|c|c|c|c|c|c|}
\hline \multicolumn{5}{|c|}{ Free fermions } & \multicolumn{6}{|c|}{ Single fusion, with $Q_{\mathrm{imp}}=\left(0, \frac{1}{2}, \frac{1}{2}\right)$} & \multirow{2}{*}{$\frac{\mathrm{NRG}}{E_{\mathrm{NRG}}}$} & \multicolumn{5}{|c|}{ Double fusion, with $Q_{\text {imp }}=\left(0, \frac{1}{2}, \frac{1}{2}\right)$} \\
\hline$q$ & $S$ & $\lambda$ & $d$ & E & $q$ & $S^{\prime}$ & $\lambda^{\prime}$ & $d^{\prime}$ & $E^{\prime}$ & $\delta E^{\prime}$ & & $q$ & $S^{\prime \prime}$ & $\lambda^{\prime \prime}$ & $\Delta$ & $\hat{O}$ \\
\hline \multirow{4}{*}{0} & \multirow{4}{*}{0} & \multirow{4}{*}{0} & \multirow{4}{*}{1} & \multirow{4}{*}{0} & \multirow{4}{*}{0} & \multirow{4}{*}{$\frac{1}{2}$} & \multirow{4}{*}{$\frac{1}{2}$} & \multirow{4}{*}{4} & \multirow{4}{*}{$\frac{3}{8}$} & \multirow{4}{*}{$\frac{1}{4}$} & \multirow{4}{*}{0.25} & \multirow{4}{*}{0} & 0 & 0 & 0 & \\
\hline & & & & & & & & & & & & & 0 & 1 & $\frac{1}{2}$ & $\boldsymbol{\Phi}_{\text {orb }}$ \\
\hline & & & & & & & & & & & & & 1 & 0 & $\frac{1}{2}$ & $\boldsymbol{\Phi}_{\mathrm{sp}}$ \\
\hline & & & & & & & & & & & & & & 1 & 1 & \\
\hline \multirow{4}{*}{ \pm 1} & \multirow{4}{*}{$\frac{1}{2}$} & \multirow{4}{*}{$\frac{1}{2}$} & \multirow{4}{*}{4} & \multirow{4}{*}{$\frac{1}{2}$} & \multirow{4}{*}{ \pm 1} & \multirow{3}{*}{0} & 0 & 1 & $\frac{1}{8}$ & 0 & 0 & & & & & \\
\hline & & & & & & & 1 & 3 & $\frac{5}{8}$ & $\frac{1}{2}$ & 0.5 & & & & & \\
\hline & & & & & & & 0 & 3 & $\frac{5}{8}$ & $\frac{1}{2}$ & 0.5 & & & & & \\
\hline & & & & & & 1 & 1 & 9 & $\frac{9}{8}$ & 1 & 0.99 & & & & & \\
\hline 0 & 1 & 0 & 3 & $\frac{1}{2}$ & 0 & $\frac{1}{2}$ & $\frac{1}{2}$ & 4 & $\frac{3}{8}$ & $\frac{1}{4}$ & 0.25 & & & & & \\
\hline 0 & 0 & 1 & 3 & $\frac{1}{2}$ & 0 & $\frac{1}{2}$ & $\frac{1}{2}$ & 4 & $\frac{3}{8}$ & $\frac{1}{4}$ & 0.25 & & & & & \\
\hline \pm 2 & 0 & 0 & 1 & $\frac{1}{2}$ & \pm 2 & $\frac{1}{2}$ & $\frac{1}{2}$ & 4 & $\frac{7}{8}$ & $\frac{3}{4}$ & 0.75 & & & & & \\
\hline \pm 2 & 1 & 0 & 1 & 1 & \pm 2 & $\frac{1}{2}$ & $\frac{1}{2}$ & 4 & $\frac{7}{8}$ & $\frac{3}{4}$ & 0.75 & & & & & \\
\hline \pm 2 & 0 & 1 & 1 & 1 & \pm 2 & $\frac{1}{2}$ & $\frac{1}{2}$ & 4 & $\frac{7}{8}$ & $\frac{3}{4}$ & 0.75 & & & & & \\
\hline \pm 2 & 1 & 1 & 1 & $\frac{3}{2}$ & \pm 2 & $\frac{1}{2}$ & $\frac{1}{2}$ & 4 & $\frac{7}{8}$ & $\frac{3}{4}$ & 0.75 & & & & & \\
\hline
\end{tabular}

spin-1/2 impurity having no orbital isospin (obtained from Ye's model by using $\lambda=\bullet$ for the impurity orbital pseudospin, and setting $K_{0}=I_{0}=0$ ).

\section{2. $I_{0} \neq 0$ : FL fixed point}

For $I_{0} \neq 0$, particle-hole symmetry is broken. Figure 8(c) shows the eigenlevel flow computed by $\mathrm{NRG}$ for $\mathbf{c}_{0}=$ $(0,0.3,0.05)$. The low-energy fixed point is a FL, as predicted by Ye. Its spectrum shows the same equidistant set of energies as the NFL spectrum of $I_{0}=0$ [Fig. 8(a)], but the degeneracies are different. This fixed point cannot be understood by simultaneous fusion in the spin and orbital sector. However, it agrees with the FL spectrum of an SU(4) Kondo model with the higher symmetry $\mathrm{U}(1)_{\mathrm{ch}} \times \mathrm{SU}(4)_{\mathrm{fl}}$, defined in analogy to the $\mathrm{SU}(6)$ Kondo model from Sec. VE2, with a flavor index $\nu=$ $1, \ldots, 4$ encoding both spin and orbital degrees of freedom. Using non-Abelian bosonization according to the $\mathrm{U}(1) \times$ $\mathrm{SU}(4)_{1} \mathrm{KM}$ algebra, the free bath spectrum can be expressed as

$$
\begin{aligned}
E(q, \lambda)= & \frac{1}{8} q^{2}+\frac{1}{5} \kappa_{4}(\lambda)+\ell \\
\kappa_{4}(\lambda)= & \frac{1}{8}\left(3 \lambda_{1}^{2}+4 \lambda_{2}^{2}+3 \lambda_{3}^{2}+4 \lambda_{1} \lambda_{2}+2 \lambda_{2} \lambda_{3}+4 \lambda_{1} \lambda_{3}\right. \\
& \left.+12 \lambda_{1}+16 \lambda_{2}+12 \lambda_{3}\right)
\end{aligned}
$$

with $\ell \in \mathbb{Z}$, where $\kappa_{4}(\lambda)$ is the quadratic Casimir for the $\lambda=\left(\lambda_{1}, \lambda_{2}, \lambda_{3}\right)$ representation of the SU(4) Lie algebra. [The contributions from the two terms of Eq. (A2) are listed in Table S8 of the Supplemental Material [54] for the lowest few $q$ and $\lambda$ values.] Combining the flavor degrees of freedom of bath and impurity, $\lambda \otimes \lambda_{\text {imp }}=\sum_{\oplus} \lambda^{\prime}$, using the

TABLE VI. Fusion table for the FL fixed point of the SU(4) Kondo model. It is structured just as Table IV for the SU(6) Kondo model, but here the free bath excitations are computed using Eqs. (A2) and Table S8 of the SM [54], and flavor fusion is performed using $\mathrm{SU}(4)_{1}$ fusion rules (Table S9 of the SM [54]). The NRG spectrum was computed for the 2 soK model with

\begin{tabular}{|c|c|c|c|c|c|c|c|c|c|}
\hline \multicolumn{4}{|c|}{ Free fermions } & \multicolumn{5}{|c|}{ Single fusion, $Q_{\mathrm{imp}}=(0, \square)$} & \multirow{2}{*}{$\frac{\mathrm{NRG}}{E_{\mathrm{NRG}}}$} \\
\hline$q$ & $\lambda$ & $d$ & $E$ & $q$ & $\lambda^{\prime}$ & $d^{\prime}$ & $E^{\prime}$ & $\delta E^{\prime}$ & \\
\hline 0 & $\bullet$ & 1 & 0 & 0 & $\square$ & 4 & $\frac{3}{8}$ & $\frac{1}{4}$ & 0.25 \\
\hline+1 & $\square$ & 4 & $\frac{1}{2}$ & +1 & $\boxminus$ & 6 & $\frac{5}{8}$ & $\frac{1}{2}$ & 0.50 \\
\hline-1 & 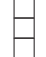 & 4 & $\frac{1}{2}$ & -1 & • & 1 & $\frac{1}{8}$ & 0 & 0 \\
\hline \pm 2 & $\square$ & 6 & 1 & \pm 2 & $\boxminus$ & 4 & $\frac{7}{8}$ & $\frac{3}{4}$ & 0.75 \\
\hline+3 & 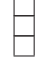 & 4 & $\frac{3}{2}$ & +3 & $\bullet$ & 1 & $\frac{9}{8}$ & 1 & 1.00 \\
\hline-3 & $\square$ & 4 & $\frac{3}{2}$ & -3 & $\boxminus$ & 6 & $\frac{13}{8}$ & $\frac{3}{2}$ & 1.50 \\
\hline
\end{tabular}
$\left(J_{0}, K_{0}, I_{0}\right)=(0,0.3,0.05)$ [Fig. 8(c)]. The single-fusion and NRG spectra agree very well (deviations $\lesssim 1 \%$ ). 
fusion rules of the $\mathrm{SU}(4)_{1} \mathrm{KM}$ algebra, we recover the $\mathrm{FL}$ fixed point spectrum found by NRG. This is shown in Table VI. In the FL regime, the spin and orbital susceptibilities scale as $\chi_{\text {sp,orb }}^{\text {imp }} \sim \omega^{1}$ [Fig. 8(d)], as expected for a Fermi liquid and predicted by Ye.

[1] A. Georges, L. de' Medici, and J. Mravlje, Strong Electronic Correlations from Hund's Coupling, Annu. Rev. Condens. Matter Phys. 4, 137 (2013).

[2] P. Werner, E. Gull, M. Troyer, and A. J. Millis, Spin Freezing Transition and Non-Fermi-Liquid Self-Energy in a Three-Orbital Model, Phys. Rev. Lett. 101, 166405 (2008).

[3] K. Haule and G. Kotliar, Coherence-Incoherence Crossover in the Normal State of Iron Oxypnictides and Importance of Hund's Rule Coupling, New J. Phys. 11, 025021 (2009).

[4] Z. P. Yin, K. Haule, and G. Kotliar, Magnetism and Charge Dynamics in Iron Pnictides, Nat. Phys. 7, 294 (2011).

[5] Z. P. Yin, K. Haule, and G. Kotliar, Kinetic Frustration and the Nature of the Magnetic and Paramagnetic States in Iron Pnictides and Iron Chalcogenides, Nat. Mater. 10, 932 (2011).

[6] L. de' Medici, J. Mravlje, and A. Georges, Janus-Faced Influence of Hund's Rule Coupling in Strongly Correlated Materials, Phys. Rev. Lett. 107, 256401 (2011).

[7] J. Mravlje, M. Aichhorn, T. Miyake, K. Haule, G. Kotliar, and A. Georges, Coherence-Incoherence Crossover and the Mass-Renormalization Puzzles in $\mathrm{Sr}_{2} \mathrm{RuO}_{4}$, Phys. Rev. Lett. 106, 096401 (2011).

[8] Z. P. Yin, K. Haule, and G. Kotliar, Fractional Power-Law Behavior and Its Origin in Iron-Chalcogenide and Ruthenate Superconductors: Insights from First-Principles Calculations, Phys. Rev. B 86, 195141 (2012).

[9] C. Aron and G. Kotliar, Analytic Theory of Hund's Metals: A Renormalization Group Perspective, Phys. Rev. B 91, 041110(R) (2015).

[10] S. Hoshino and P. Werner, Superconductivity from Emerging Magnetic Moments, Phys. Rev. Lett. 115, 247001 (2015).

[11] H. T. Dang, J. Mravlje, A. Georges, and A. J. Millis, Electronic Correlations, Magnetism, and Hund's Rule Coupling in the Ruthenium Perovskites $\mathrm{SrRuO}_{3}$ and $\mathrm{CaRuO}_{3}$, Phys. Rev. B 91, 195149 (2015).

[12] J. Mravlje and A. Georges, Thermopower and Entropy: Lessons from $\mathrm{Sr}_{2} \mathrm{RuO}_{4}$, Phys. Rev. Lett. 117, 036401 (2016).

[13] M. Zingl, J. Mravlje, M. Aichhorn, O. Parcollet, and A. Georges, Hall Coefficient Signals Orbital Differentiation in the Hund's Metal $\mathrm{Sr}_{2} \mathrm{RuO}_{4}$, npj Quantum Mater. 4, 35 (2019).

[14] T.-H. Lee, A. Chubukov, H. Miao, and G. Kotliar, Pairing Mechanism in Hund's Metal Superconductors and the Universality of the Superconducting Gap to Critical Temperature Ratio, Phys. Rev. Lett. 121, 187003 (2018).

[15] M. Yi, D. H. Lu, R. Yu, S. C. Riggs, J.-H. Chu, B. Lv, Z. K. Liu, M. Lu, Y.-T. Cui, M. Hashimoto, S.-K. Mo, Z. Hussain, C. W. Chu, I. R. Fisher, Q. Si, and Z.-X. Shen, Observation of Temperature-Induced Crossover to an Orbital-Selective Mott Phase in $A_{x} \mathrm{Fe}_{2-y} \mathrm{Se}_{2}(A=\mathrm{K}, \mathrm{Rb})$ Superconductors, Phys. Rev. Lett. 110, 067003 (2013).
[16] F. Hardy, A. E. Böhmer, D. Aoki, P. Burger, T. Wolf, P. Schweiss, R. Heid, P. Adelmann, Y. X. Yao, G. Kotliar, J. Schmalian, and C. Meingast, Evidence of Strong Correlations and Coherence-Incoherence Crossover in the Iron Pnictide Superconductor $\mathrm{KFe}_{2} \mathrm{As}_{2}$, Phys. Rev. Lett. 111, 027002 (2013).

[17] A. Horvat, R. Žitko, and J. Mravlje, Low-Energy Physics of Three-Orbital Impurity Model with Kanamori Interaction, Phys. Rev. B 94, 165140 (2016).

[18] A. Tamai, M. Zingl, E. Rozbicki, E. Cappelli, S. Riccò, A. de la Torre, S. M. Walker, F. Y. Bruno, P. D. C. King, W. Meevasana, M. Shi, M. Radović, N. C. Plumb, A. S. Gibbs, A. P. Mackenzie, C. Berthod, H. U. R. Strand, M. Kim, A. Georges, and F. Baumberger, High-Resolution Photoemission on $\mathrm{Sr}_{2} \mathrm{RuO}_{4}$ Reveals Correlation-Enhanced Effective Spin-Orbit Coupling and Dominantly Local Self-Energies, Phys. Rev. X 9, 021048 (2019).

[19] P. Werner and S. Hoshino, Nickelate SuperconductorsMultiorbital Nature and Spin Freezing, Phys. Rev. B 101, 041104(R) (2020).

[20] P. Coleman, Y. Komijani, and E. König, The Triplet Resonating Valence Bond State and Superconductivity in Hund's Metals, arXiv:1910.03168 [Phys. Rev. Lett. (to be published)].

[21] X. Chen, I. Krivenko, M. B. Stone, A. I. Kolesnikov, T. Wolf, D. Reznik, K. S. Bedell, F. Lechermann, and S. D. Wilson, Unconventional Hund Metal in MnSi, arXiv:1909.11195.

[22] K. M. Stadler, Z. P. Yin, J. von Delft, G. Kotliar, and A. Weichselbaum, Dynamical Mean-Field Theory Plus Numerical Renormalization-Group Study of Spin-Orbital Separation in a Three-Band Hund Metal, Phys. Rev. Lett. 115, 136401 (2015).

[23] K. M. Stadler, G. Kotliar, A. Weichselbaum, and J. von Delft, Hundness versus Mottness in a Three-Band HubbardHund Model: On the Origin of Strong Correlations in Hund Metals, Ann. Phys. (Amsterdam) 405, 365 (2019).

[24] X. Deng, K. M. Stadler, K. Haule, A. Weichselbaum, J. von Delft, and G. Kotliar, Signatures of Mottness and Hundness in Archetypal Correlated Metals, Nat. Commun. 10, 2721 (2019).

[25] S. Akhanjee and A. M. Tsvelik, Analytically Tractable Model of Bad Metals, Phys. Rev. B 87, 195137 (2013).

[26] J. Kondo, Resistance Minimum in Dilute Magnetic Alloys, Prog. Theor. Phys. 32, 37 (1964).

[27] P. W. Anderson, A Poor Man's Derivation of Scaling Laws for the Kondo Problem, J. Phys. C 3, 2436 (1970).

[28] K. G. Wilson, The Renormalization Group: Critical Phenomena and the Kondo Problem, Rev. Mod. Phys. 47, 773 (1975).

[29] J. R. Schrieffer, The Kondo Effect-The Link between Magnetic and Nonmagnetic Impurities in Metals?, J. Appl. Phys. 38, 1143 (1967).

[30] M. D. Daybell and W. A. Steyert, Localized Magnetic Impurity States in Metals: Some Experimental Relationships, Rev. Mod. Phys. 40, 380 (1968).

[31] B. Coqblin and J. R. Schrieffer, Exchange Interaction in Alloys with Cerium Impurities, Phys. Rev. 185, 847 (1969).

[32] I. Okada and K. Yosida, Singlet Ground State of the Localized d-Electrons Coupled with Conduction Electrons in Metals, Prog. Theor. Phys. 49, 1483 (1973). 
[33] P. Nozières and A. Blandin, Kondo Effect in Real Metals, J. Phys. (Paris) 41, 193 (1980).

[34] N. Andrei and C. Destri, Solution of the Multichannel Kondo Problem, Phys. Rev. Lett. 52, 364 (1984).

[35] A. M. Tsvelik and P. B. Wiegmann, Solution of the n-Channel Kondo Problem (Scaling and Integrability), Z. Phys. B 54, 201 (1984).

[36] A. M. Tsvelik, The Thermodynamics of Multichannel Kondo Problem, J. Phys. C 18, 159 (1985).

[37] A. M. Tsvelik and P. B. Wiegmann, Exact Solution of the Multichannel Kondo Problem, Scaling, and Integrability, J. Stat. Phys. 38, 125 (1985).

[38] N. Andrei and A. Jerez, Fermi- and Non-Fermi-Liquid Behavior in the Anisotropic Multichannel Kondo Model: Bethe Ansatz Solution, Phys. Rev. Lett. 74, 4507 (1995).

[39] A. Jerez, N. Andrei, and G. Zaránd, Solution of the Multichannel Coqblin-Schrieffer Impurity Model and Application to Multilevel Systems, Phys. Rev. B 58, 3814 (1998).

[40] D. M. Cragg, P. Lloyd, and P. Nozieres, On the Ground States of Some $s$-d Exchange Kondo Hamiltonians, J. Phys. C 13, 803 (1980).

[41] H. B. Pang and D. L. Cox, Stability of the Fixed Point of the Two-Channel Kondo Hamiltonian, Phys. Rev. B 44, 9454 (1991).

[42] I. Affleck, A Current Algebra Approach to the Kondo Effect, Nucl. Phys. B336, 517 (1990).

[43] I. Affleck and A. W. W. Ludwig, The Kondo Effect, Conformal Field Theory and Fusion Rules, Nucl. Phys. B352, 849 (1991).

[44] I. Affleck and A. W. W Ludwig, Critical Theory of Overscreened Kondo Fixed Points, Nucl. Phys. B360, 641 (1991).

[45] I. Affleck and A. W. W. Ludwig, Exact Conformal-FieldTheory Results on the Multichannel Kondo Effect: SingleFermion Green's Function, Self-Energy, and Resistivity, Phys. Rev. B 48, 7297 (1993).

[46] A.W.W. Ludwig and I. Affleck, Exact ConformalField-Theory Results on the Multi-Channel Kondo Effect: Asymptotic Three-Dimensional Space- and Time-Dependent Multi-Point and Many-Particle Green's Functions, Nucl. Phys. B428, 545 (1994).

[47] K. M. Stadler, A Model Study of Strong Correlations in Hund Metals. The Numerical Renormalization Group as Efficient Multi-Band Impurity Solver for Dynamical Mean-Field Theory, Ph.D. thesis, Ludwig-MaximiliansUniversität München, 2019.

[48] J. Ye, Solution of the Two-Channel Spin-Flavor Kondo Model, Phys. Rev. B 56, R489 (1997).

[49] Y. Wang, E. Walter, S.-S. B. Lee, K. M. Stadler, J. von Delft, A. Weichselbaum, and G. Kotliar, Global Phase Diagram of a Spin-Orbital Kondo Impurity Model and the Suppression of Fermi-Liquid Scale, Phys. Rev. Lett. 124, 136406 (2020).
[50] A. Weichselbaum, Non-Abelian Symmetries in Tensor Networks: A Quantum Symmetry Space Approach, Ann. Phys. (Amsterdam) 327, 2972 (2012).

[51] A. Weichselbaum, Tensor Networks and the Numerical Renormalization Group, Phys. Rev. B 86, 245124 (2012).

[52] A. Weichselbaum and J. von Delft, Sum-Rule Conserving Spectral Functions from the Numerical Renormalization Group, Phys. Rev. Lett. 99, 076402 (2007).

[53] S.-S. B. Lee and A. Weichselbaum, Adaptive Broadening to Improve Spectral Resolution in the Numerical Renormalization Group, Phys. Rev. B 94, 235127 (2016).

[54] See Supplemental Material at http://link.aps.org/ supplemental/10.1103/PhysRevX.10.031052 for tables presenting detailed fusion rules and contributions to lowenergy spectra, which includes Refs. [55,56].

[55] C. J. Cummins, $s u(n)$ and $s p(2 n)$ WZW Fusion Rules, J. Phys. A 24, 391 (1991).

[56] P. Di Francesco, P. Mathieu, and D. Sénéchal, Conformal Field Theory (Springer, New York, 1997).

[57] J. von Delft, 2-Channel Kondo Scaling in Metal Nanoconstrictions-A Conformal Field Theory Calculation of Scaling Function, Ph.D. Thesis, Cornell University, 1995.

[58] A. W. W. Ludwig, Field Theory Approach to Critical Quantum Impurity Problems and Applications to the Multichannel Kondo Effect, Int. J. Mod. Phys. B 08, 347 (1994).

[59] J. L. Cardy, Conformal Invariance and Surface Critical Behavior, Nucl. Phys. B240, 514 (1984).

[60] The quantum numbers of $\tilde{\Psi}_{\text {orb }}$ differ from those of $\boldsymbol{\Phi}_{\text {orb }}$ (and hence also of $\mathbf{T}, \mathbf{J}_{\mathrm{orb}}$ ), since the same is true for their parent states, reflecting the fact that the latter undergo a true level crossing. Ditto for $\tilde{\Psi}_{\mathrm{sp}}$ versus $\boldsymbol{\Phi}_{\mathrm{sp}}$ (and $\mathbf{S}, \mathbf{J}_{\mathrm{sp}}$ ). In contrast to $\mathbf{S} \cdot \boldsymbol{\Phi}_{\text {sp }}$, it is hence not possible to couple $\mathbf{S}$ and $\tilde{\Psi}_{\text {sp }}$ to a spin singlet, needed for computing $\chi_{\mathrm{sp}}^{\text {imp,bath }}$. Instead, we speculate that the role of $\left(\int d t^{\prime} S^{a} \Phi_{\mathrm{sp}}^{a}\right)^{2}$ could be taken, e.g., by $\int d t^{\prime} S^{a}\left(\tilde{\Psi}_{\mathrm{sp}}^{\dagger}\right)_{\sigma} \int d t^{\prime \prime} S^{a}\left(\tilde{\Psi}_{\mathrm{sp}}\right)_{\sigma}$.

[61] I. Affleck, Conformal Field Theory Approach to the Kondo Effect, Acta Phys. Polon. B 26, 1869 (1995), https://www .actaphys.uj.edu.pl/R/26/12/1869/pdf.

[62] J. Fuchs and C. Schweigert, Symmetries, Lie Algebras and Representations: A Graduate Course for Physicists (Cambridge University Press, 1997), p. 335, Eq. (19.14).

[63] T. A. Costi, Kondo Effect in a Magnetic Field and the Magnetoresistivity of Kondo Alloys, Phys. Rev. Lett. 85, 1504 (2000).

[64] A. A. Khajetoorians, M. Valentyuk, M. Steinbrecher, T. Schlenk, A. Shick, J. Kolorenc, A. I. Lichtenstein, T. O. Wehling, R. Wiesendanger, and J. Wiebe, Tuning Emergent Magnetism in a Hund's Impurity, Nat. Nanotechnol. 10, 958 (2015).

[65] A. Horvat, R. Žitko, and J. Mravlje, Non-Fermi-Liquid Fixed Point in Multi-Orbital Kondo Impurity Model Relevant for Hund's Metals, arXiv:1907.07100. 